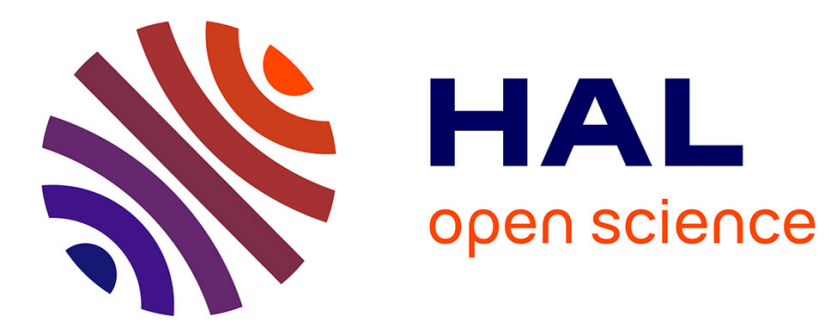

\title{
Horizontal drying fronts in films of colloidal dispersions: influence of hydrostatic pressure and collective diffusion
}

\author{
M. Nassar, A. Gromer, D. Favier, F. Thalmann, P. Hébraud, Y. Holl
}

\section{To cite this version:}

M. Nassar, A. Gromer, D. Favier, F. Thalmann, P. Hébraud, et al.. Horizontal drying fronts in films of colloidal dispersions: influence of hydrostatic pressure and collective diffusion. Soft Matter, 2017, 13 (48), pp.9162-9173. 10.1039/C7SM01334E . hal-03586510

\section{HAL Id: hal-03586510 https://hal.science/hal-03586510}

Submitted on 23 Feb 2022

HAL is a multi-disciplinary open access archive for the deposit and dissemination of scientific research documents, whether they are published or not. The documents may come from teaching and research institutions in France or abroad, or from public or private research centers.
L'archive ouverte pluridisciplinaire HAL, est destinée au dépôt et à la diffusion de documents scientifiques de niveau recherche, publiés ou non, émanant des établissements d'enseignement et de recherche français ou étrangers, des laboratoires publics ou privés. 


\title{
Horizontal drying fronts in films of colloidal dispersions: influence of hydrostatic pressure and collective diffusion
}

\author{
M. Nassar, ${ }^{1}$ A. Gromer, ${ }^{1}$ D. Favier, ${ }^{1}$ F. Thalmann, ${ }^{1}$ P. Hébraud,${ }^{2}$ Y. Holl ${ }^{1, *}$ \\ 1. CNRS-ICS \& Université de Strasbourg \\ 23, rue du Loess BP 84047 \\ 67034 Strasbourg Cedex 2 \\ France \\ 2. IPCMS, 23 rue du Loess BP 43 \\ 67034 STRASBOURG Cedex 2 \\ France \\ *yves.holl@unistra.fr
}

\begin{abstract}
The origin and time evolution of heterogeneities in drying colloidal films is still a matter of debate. In this work, we studied the behaviour of horizontal drying fronts in a 1D configuration. The effects of hydrostatic pressure and collective diffusion of charged particles, neglected so far, were introduced. We made use of the new simulation tool based on cellular automata we recently presented (Langmuir 2015 \& 2017). To check the simulation results, measurements of film profiles in the wet state and drying front velocities were performed with silica colloids. It was shown that taking hydrostatic pressure into account much improves agreement between theory and experiment. On the other hand, the simulation showed that collective diffusion slows down the drying fronts, even more when the Debye length is increased. This latter effect remains to be checked experimentally. This work opens the way to further improvements of theory and simulation, notably 2D and 3D simulations.
\end{abstract}




\section{Introduction}

Drying mechanisms of volatile liquids containing non-volatile compounds (essentially particles but also salts and high molar weight molecules) is the research topic of many groups worldwide. The reason for this interest is twofold: the vast diversity of domains making use of colloid drying and the extreme complexity of phenomena involved. Applications include forming templates on solid surfaces, ink-jet printing, spraying of pesticides, micro/nano material fabrication, coatings and adhesives, biochemical assays, deposition of DNA/RNA micro-arrays, manufacture of novel optical and electronic materials, as mentioned by Erbil in his review on deposits formed by evaporation of dispersions. ${ }^{1}$ Drying mechanisms consist of thermal, mechanical and transport phenomena in complex interplay over large time and space scales. ${ }^{2,3}$

A general characteristic of colloid drying, with only few exceptions, is heterogeneity: soon appear drier regions on top and on edges of the film or drop, separated from wetter regions by more or less diffuse interfaces called "drying fronts" moving toward the substrate and the centre at different speeds. The front parallel to the substrate and moving in the vertical direction is much slower than the fronts perpendicular to the substrate moving laterally. These fronts were observed a long time ago ${ }^{4,5,6}$ and nicely visualized by cryomicroscopy by Scriven et al. ${ }^{7}$ The drying fronts are associated with transport phenomena.

Transport of matter, by convection and diffusion, occurs in the vertical and/or in the horizontal directions. In the vertical direction, convection is due to the liquid / air interface moving downward due to drying. ${ }^{8}$ In the horizontal directions, convection is generated by pressure gradients in the bulk of the film ${ }^{9}$ or by surface tension gradients (Marangoni flow). ${ }^{10}$ Convection of particles and other components of the colloid has important consequences in terms of film morphology ${ }^{11}$ and distribution of additives, ${ }^{12,13,14}$ which, in turn, strongly influence application properties. An interesting example is stratification in the vertical direction according to particle sizes ${ }^{15,16}$ or charges. ${ }^{17}$ A technique based on drying driven convection called "Evaporative lithography" has been developed in order to manipulate the final film topography. ${ }^{18,19,20}$

Let us now concentrate on lateral drying fronts and associated convection (two schemes in the Supplementary Information help the reader to better understand the following considerations). The best known example of such a phenomenon is the coffee stain left after drying, popularized by a paper in Nature by Deegan et al. ${ }^{21}$ In this case, the sample is in the form of a drop of a very dilute colloid and, importantly, the contact line is pinned onto the substrate during the whole drying process. According to Deegan et al., convection carrying 
particles from the centre to the edge is due to a higher evaporation rate at the edge and to the surface tension of the drop inducing a spherical cap shape. ${ }^{22}$ This condition of a higher evaporation rate at the edge does not appear in all models of lateral convection. ${ }^{9,23}$ In films, similar behaviours can be observed. There are many examples in the field of latex (polymer colloid) drying. ${ }^{24}$ Winnik and Feng proposed a drying process where propagation of a lateral front in films was due to evaporation from a region at the edge where particles had concentrated to form a close packed structure. ${ }^{25}$ Routh and Russel mathematically elaborated on this picture. ${ }^{9}$ Their model was based on the following scenario. They first considered colloids with nondeformable particles. Casting the colloid on an appropriate substrate results in a contact angle less than $90^{\circ}$ because, otherwise, dewetting would occur. In the region near the edge, the fluid will also present a convex curvature leading to a pressure gradient (originating from Laplace pressure) in the lateral direction. The edge will be thinner than the centre of the deposit and, for this reason, even without considering a faster evaporation rate at the edge, it will concentrate faster than the centre and sooner reach the state of particle close packing. The close packed area (which can be called a "wet gel"), where water still evaporates, exerts a capillary aspiration on the nearby fluid. The combination of flows due to aspiration and curvature leads to a profile of the deposit, the shape and time evolution of which Routh and Russel could calculate (details of this calculation will be given in next sections). The profile presents a bulge close to the contact line, higher than the level at the film centre, and oscillations. Knowing the deposit profile at each time together with the evaporation rate, allowed them to calculate the velocity field in the fluid, the particle concentration profile and the speed of the inward moving interface between the close packed and the fluid areas in the film (called "compaction front" or sometimes "particle front"). A quick look at Fig. 3 below and Fig. S2 in the Supplementary Information will help a better understanding of the last few sentences. Some articles were devoted to a more or less successful experimental validation of the Routh and Russel model. ${ }^{26,27}$

This paper deals with lateral heterogeneities ${ }^{28}$ due to transport of matter by convection and diffusion, accompanying drying. On one hand, if we assume that Laplace pressure, due to the curvature of the air/latex interface, controls the lateral flow, an accumulation of matter is obtained close to the compaction front, leading to spatial oscillations of the surface whose first maximum can be higher than the fluid phase far from the front. On the other hand, in the limit where the pressure is dominated by the hydrostatic pressure, the height of the air/fluid interface increases monotonously from the contact line to the centre of the deposit with an exponential behaviour. We will thus devise a model, starting from the Routh and Russel description of 
evaporation, in which we include the contributions of the hydrostatic pressure and the Laplace pressure as well as the collective diffusion of interacting particles. We will see that this leads to very different film height profiles and different front velocities that we will compare to experimental measurements.

In a first part, we introduce the theoretical description of film drying, including the hydrostatic pressure and taking into account the cooperative diffusion of the colloidal particles. Then, we explicit our simulation approach (already presented and used in two preceding papers $\left.{ }^{29,30}\right)$ : it consists of dividing the drying system in space filing cells exchanging matter according to physical laws of increasing complexity. It is inspired by cellular automata ${ }^{31}$ and uses finite difference methods. At each time step, the flux of matter in and out one cell depends on the state of its direct neighbours and obeys local rules. The evolution of the whole system is described by integrating the changing states of all cells over space and time. This kind of simulation allows an easier handling of the heterogeneous nature of drying colloids. It has also the advantage of progressivity in increasing the complexity of treated cases in terms of dimensionality (1, 2 and 3D), number of constituents taken into account and nature of the considered physical laws (exchange of matter, and subsequently heat and stress). Numerical results are then compared to experimental data of drying of suspension of silica particles.

\section{Theoretical considerations}

Routh and Russel $^{9}$ (RR) designed a theoretical model for horizontal fronts propagating through drying colloidal films. Here we summarize the main features of this model. A semi-infinite film is considered extending in the direction of $x>0$, with $x=0$ corresponding to the film edge. The film is divided into a wet gel domain, which extends from $\mathrm{x}=0$ up to $\mathrm{x}=\mathrm{x}_{\mathrm{f}}$, the position of the compaction front, and a fluid domain, for $\mathrm{x}>\mathrm{x}_{\mathrm{f}}$. Vertical inhomogeneities in the particle volume fraction are neglected. Using the Navier-Stokes equation and the lubrication approximation, vertically averaged equations for particle and fluid conservation can be derived in the fluid and wet gel domains (see Table below). 


\begin{tabular}{|l|l|l|}
\hline & Gel domain & Fluid domain \\
\hline Particle conservation & $\frac{\partial \bar{h} \phi}{\partial \bar{t}}=0$ & $\frac{\partial \bar{h} \phi}{\partial \bar{t}}+\frac{\partial \phi \bar{h} \bar{u}_{x}}{\partial \bar{x}}=0$ \\
\hline Fluid conservation & $1+\frac{\partial \bar{h} \bar{u}_{x}(1-\phi)}{\partial \bar{x}}=0$ & $1+\frac{\partial \bar{h}}{\partial \bar{t}}+\frac{\partial \bar{h} \bar{u}_{x}}{\partial \bar{x}}=0$ \\
\hline Fluid velocity & $\bar{u}_{x}=-\frac{\partial \bar{p}}{\partial \bar{x}}$ & $\overline{u_{x}}=\bar{h}^{2} \frac{\partial^{3} \bar{h}}{\partial \bar{x}^{3}}$ \\
\hline
\end{tabular}

In these equations, $\phi$ is the particle volume fraction, $\mathrm{u}_{\mathrm{x}}$ is the fluid velocity in direction $\mathrm{x}$, the film height (h) is scaled on the initial height: $H_{0}$, time (t) is scaled on a characteristic time: $\frac{H_{O}}{\dot{E}}$, with $\dot{E}$ the drying rate, and horizontal distances (x) are scaled on a characteristic length that RR called the "capillary length", defined in reference 9 as the length for which the flow due to surface tension is balanced by that caused by evaporation: $L_{c a p}=H_{0}\left(\frac{\gamma}{3 \eta_{0} \dot{E}}\right)^{1 / 4}$ with $\gamma=$ waterair interfacial tension and $\eta_{0}$ the zero shear viscosity of the dispersion. Finally, pressure is scaled on a characteristic pressure: $P=\frac{\eta_{0} L_{c a p}^{2} \dot{E}}{H_{0}^{3}}$. To calculate scaled quantities, $H_{0}$ was given the desired value (700 $\mu \mathrm{m}$ when experimental data had to be scaled), $\dot{E}$ was measured by gravimetry (see below) at $1.6 \times 10^{-8} \mathrm{~m} / \mathrm{s}, \gamma=70 \mathrm{~mJ} / \mathrm{m}^{2}$, a standard literature value for water at room temperature, and $\eta_{0}$ was given the value of 1 Pa.s like in reference 9.

The conservation equations involve the fluid velocity, which is calculated using a different expression in the gel and fluid domains: in the gel domain, the velocity is proportional to the pressure gradient (Darcy law), whereas in the fluid domain, it involves the third derivative of the film height (gradient of the Laplace pressure).

In the case of totally non deformable particles, the film height in the gel is independent of time and the volume fraction is equal to $\phi_{\max }$ (close packed network). Integrating the conservation equation of the fluid in the gel domain allows one to calculate the pressure and the fluid velocity in the gel.

The film height, volume fraction and dispersion velocity in the fluid domain can be calculated by integrating the equations from the fluid domain using the condition that, near the compaction front, the velocity of the dispersion must be equal to the velocity of the solvent on the wet gel side multiplied by $\left(1-\phi_{\max }\right)$. Then, a particle balance allows one to calculate the 
advancement of the compaction front knowing the particle volume fraction and dispersion velocity near the front (on the fluid domain side): $\frac{d \bar{x}_{f}}{d t}=\frac{-\phi\left(\bar{x}_{f}\right) \bar{u}_{x}\left(\bar{x}_{f}\right)\left(1-\phi_{\max }\right)}{\phi_{\max }-\phi\left(\bar{x}_{f}\right)}$.

As the wet gel domain grows in size, the pressure differential across it, $\Delta \bar{p}$, increases. When it reaches the maximum capillary pressure sustainable by the particle network, the pressure becomes insufficient to bring water up to the edge and the water front appears. The advancement of the water front can be calculated by using the velocity of the solvent at that position.

In this work, thermal effects ${ }^{32}$ were neglected with the argument that only thin deposits are considered in which thermal equilibrium is reached rapidly. The drying process was considered up to the time when close packing is reached in the whole film. For simplicity, we assume an infinite capillary pressure (thus, no water front appears in the gel domain). In this case, the fluid velocity near the compaction front, on the gel domain side, is simply equal to: $\frac{\bar{x}_{f}}{\bar{h}\left(1-\phi_{\max }\right)}$ (the fluid velocity near the compaction front, on the gel domain side, is equal to the evaporation rate from the packed region). Finally, hydrostatic pressure is taken into account when calculating the fluid velocity in the fluid domain, and collective diffusion in the particle conservation equation.

Hence: $\bar{u}_{x}=\bar{h}^{2}\left(\frac{\partial^{3} \bar{h}}{\partial \bar{x}^{3}}-\left(\frac{L_{c a p}}{L_{g}}\right)^{2} \frac{\partial \bar{h}}{\partial \bar{x}}\right)$ with: $L_{g}=\sqrt{\frac{\gamma}{\rho g}}$ the capillary length in the usual sense, where surface tension is balanced by gravity, ${ }^{33} \rho$ being the density of the dispersion and $g$ the gravitational acceleration. This expression for the fluid velocity is easy to demonstrate since: $u_{x}=-\frac{h^{2}}{3 \eta_{0}} \frac{\partial p}{\partial x}$ within the lubrication approximation. A detailed demonstration of the expression for the fluid velocity is given in the Electronic Supplementary Information section.

In their work, RR neglected horizontal diffusion. Integrating a local particle conservation equation over the film thickness while assuming a uniform vertical concentration led them to the particle conservation equation seen in Table 1. If horizontal diffusion is not neglected (but the vertical concentration is still assumed uniform), we obtain instead:

$\frac{\partial \bar{h} \phi}{\partial \bar{t}}+\frac{\partial}{\partial \bar{x}}\left(\phi \bar{h} \bar{u}_{x}-\bar{h} \bar{D} \frac{\partial \phi}{\partial \bar{x}}\right)=0$ with: $\bar{D}=\left(\frac{H_{0}}{\dot{E} L_{c a p}^{2}}\right) D_{\text {coop }}$ and $D_{\text {coop }}=D_{0} K(\phi) \mathrm{d}[\phi Z(\phi)] / d \phi$ $D_{0}$ is the individual diffusion coefficient of a particle (Stokes - Einstein), $K(\phi)$ is the sedimentation coefficient, calculated as in Ref. 34, and $Z(\phi)$ the compressibility factor. 
$K(\phi)=\frac{1-\frac{3}{2} \phi^{1 / 3}+\frac{3}{2} \phi^{5 / 3}-\phi^{2}}{1+\frac{2}{3} \phi^{5 / 3}}$ and $Z(\phi)=\frac{1}{\phi_{\max }-\phi}$

Rigorously, this description should be modified by taking into account the convection of the particles due to velocity gradients. This has been done by Taylor in the case of pipe flow $^{35}$ and we propose a similar analysis of the diffusion in a planar film geometry, in the Supplementary Information section. From this analysis, we conclude that convection introduces a correction to the numerical value of the diffusion coefficient, similarly to the pipe flow situation and we will not take this effect into account in the rest of our study.

\section{Program description}

Details about the way equations of theoretical origin were implemented in a computer program were described in length in our previous articles. ${ }^{29,30}$ Let us briefly recall the main characteristics of our software. The film is divided in $\mathrm{N}$ rectangular cells of width $\mathrm{dx}$ along the horizontal $\mathrm{x}$ axis. The cells and their components (here only rigid particles and water) are represented by classes that have their own variables and functions. The components have specified evolution rules that act locally and are of two types: transport rules and update rules. Transport rules deal with exchanges of matter between a cell and its immediate neighbours and are based on the particle and fluid conservation equations presented in the previous section. Update rules act on changing the component state variables in each cell. In one time step, the transport rules of all components are applied before applying the update rules, ensuring that the system evolution is synchronized. Once a cell in the liquid state reaches the maximum particle volume fraction, an update rule changes the state of the cell to wet gel. Thus, the compaction front moves by jumping from one cell boundary to the next. In the calculations used in this work, the colloid film was divided into 60 to 120 cells and the time step was chosen in order to insure the stability of the integration method. Initially, the horizontal distribution of particles was assumed to be homogeneous and the height equal to $\mathrm{Ho}$, except at the edge which we assumed rounded over the length $0.5 L_{c a p}$.

Alternatively, a purely numerical approach could also be used to solve the 1D model equations described in the above theoretical section with finite difference methods and an explicit scheme. In this case, we assumed that a small gel region of size: $\bar{x}_{g e l}=0.0001$ was formed initially at the film edge. Every time step, the compaction front moved and the fluid domain was remeshed (the values at the new nodes were determined by linear interpolation). 
It was checked that the two approaches, this numerical calculation and the cellular simulation described above, led to identical results.

\section{Experimental}

\section{Silica dispersions and film formation}

A surfactant free, model colloid with monodisperse, non-deformable silica particles in water was used. Silica particles (nominal diameter: $250 \mathrm{~nm}$ ) were purchased in form of powder from AngströmSphere and used as received. Mean particle size, particle size distribution and zeta potential were determined by Dynamic Light Scattering using a Zetasizer 3000HS from Malvern Instrument. Results are shown in Fig. 1. The size distribution was narrow, centred at $270 \mathrm{~nm}$, with a standard deviation of $58 \mathrm{~nm}$. The polydispersity index, defined as the square of the ratio of standard deviation over mean, was 0.045 . The measured zeta potential was $-45 \mathrm{mV}$ at a spontaneous $\mathrm{pH}$ of 9 . Due to dissolution of silicates in the solution, ${ }^{36}$ the Debye length is smaller than in pure water at $\mathrm{pH}=9$. We estimated the concentration of the silicate ions at $2.10^{-}$ ${ }^{4} \mathrm{M}$, leading to a Debye length of $40 \mathrm{~nm}$. 

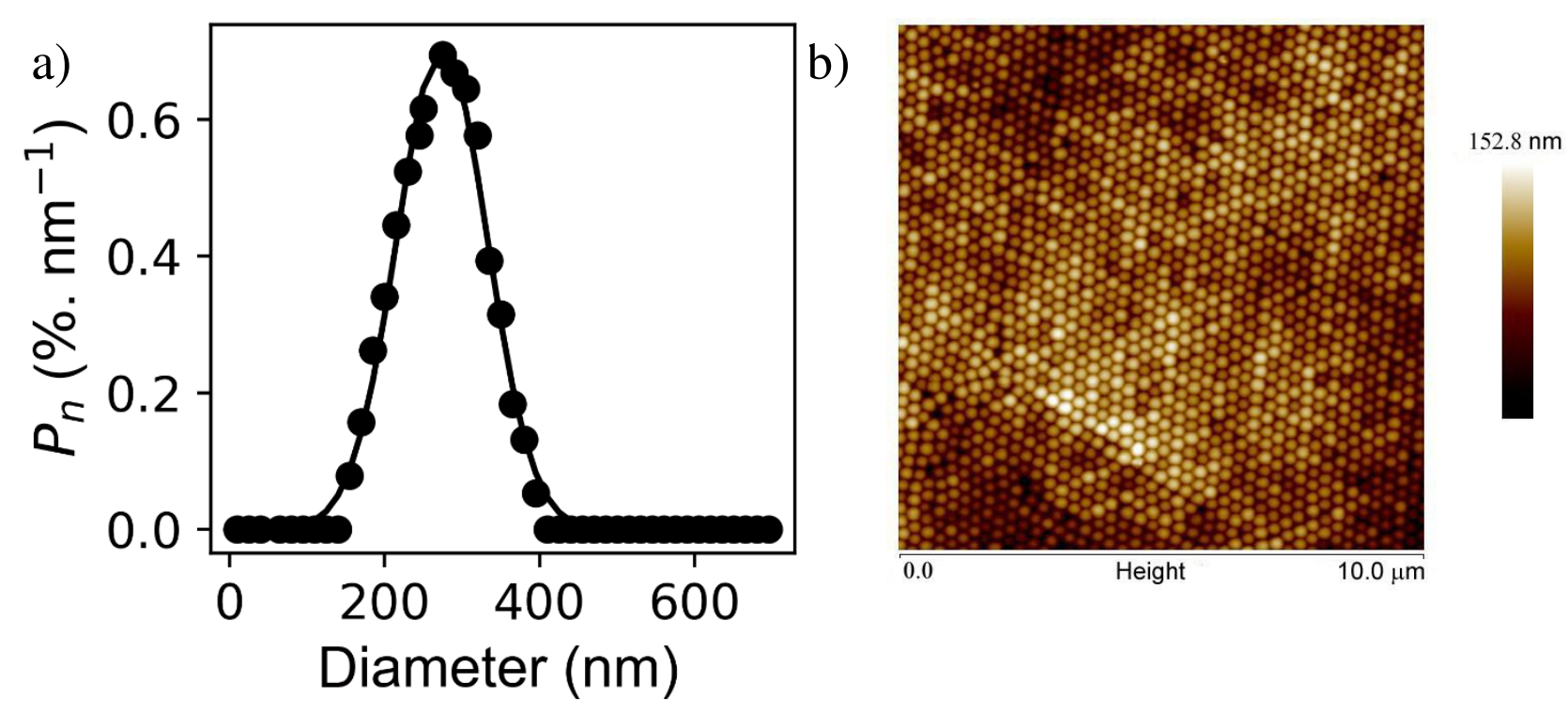

c)

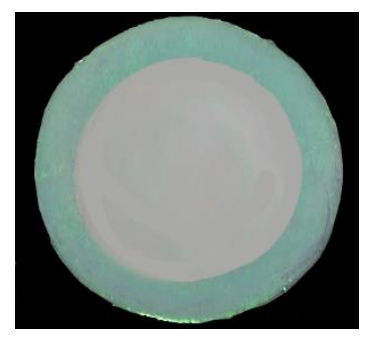

Wet gel Fluid dispersion

d)

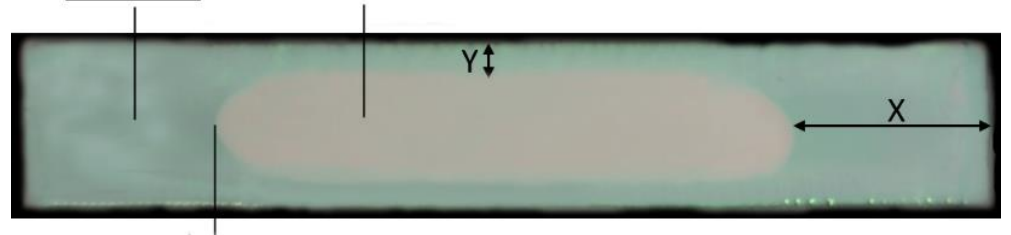

Compaction front

Fig. 1 Colloid of silica particles. Diameter $270 \mathrm{~nm}$. a) Particle size distribution of the silica particles, as measured by Dynamic Light Scattering. The average diameter was $270 \mathrm{~nm}$ and the polydispersity index was 0.045 . Continuous line: adjustment of the distribution with a Gaussian distribution of width $58 \mathrm{~nm}$. b) AFM image of the dry deposit. c) and d) Digital photographs of deposits of various shapes and sizes during drying. c) Circle, diameter $4 \mathrm{~cm}$. d) Rectangle $120 \mathrm{~mm} \times 20 \mathrm{~mm}$, the distance y was the one of interest.

Prior to film preparation, the particles were dispersed in water and sonicated with a tip sonicator for 3 minutes. The initial volume fraction in our experiments was 0.3. The prepared colloid showed no macroscopic aggregation. To ensure that our system was stable, not only at the macroscopic level, DLS was used immediately after the preparation of the suspension and eight hours later, a time significantly longer than the longest drying time. No increase of the average particle size was noticed.

Films were prepared on glass slides of different shapes and sizes: a square of sides of 53 $\mathrm{mm}$; a circle of diameter $6 \mathrm{~cm}$; a rectangle of dimensions $120 \mathrm{~mm}$ x $20 \mathrm{~mm}$; cleaned overnight in $\mathrm{H}_{2} \mathrm{SO}_{4}$ bath, rinsed in water and then in ethanol. A thin layer of polytetrafluoroethylene 
(PTFE) (Sprayflon ${ }^{\circledR}$, Roth) was sprayed around the edges of the slides over a width of $3 \mathrm{~mm}$ creating a non wettable rim that confined the dispersion on a known area in the central part of the substrate. An adequate volume of dispersion was deposited on the PTFE free part of the slide, ensuring the same film thickness of $700 \mu \mathrm{m}$ for all samples. Drying took place under controlled conditions in a Plexiglas box: $23 \pm 2^{\circ} \mathrm{C}, 75 \pm 2 \% \mathrm{RH}$, still air. These conditions minimized film cracking, at least at the beginning. ${ }^{37,38}$ Photographs of samples during drying are shown in Fig. 1 c) and d) and Fig. 2 a). The pink central part is fluid whereas the greenish area corresponds to a wet gel (particles in a close packed state with water in the interstices). The compaction front is at the interface between the two domains. The colours are due to the narrow particle size distribution of the colloid leading to visible light scattering. It was checked on a control sample that the central part was fluid and the edge side was solid like by touching the surface with a needle. It was also clear that small defects visible to the naked eye were mobile in the centre but fix on the edge.

\section{Gravimetry and image processing}

The weight loss of the film during drying was recorded with a digital microbalance (Mettler Toledo ME103E, precision 0.001g). Drying times could then be measured within an error range of $\pm 2.0 \%$, allowing us to determine the drying rate of the suspensions. In the standard conditions and the system defined above, the drying rate was $1.6 \times 10^{-8} \mathrm{~m} / \mathrm{s}$. Simultaneously, a video camera recorded the film optical changes during drying. Images were captured with a digital camera (Sony Handycam ${ }^{\circledR} 4 \mathrm{~K}$ AXP33) operating at the standard frequency of 24 images per second. Taking into account the low average velocity of the compaction fronts (around $0.04 \mathrm{~mm} / \mathrm{min}$ ) this was largely sufficient. The displacements and velocities of the horizontal compaction fronts were measured by image processing (ImageJ). The considered front was the one in the y direction (perpendicular to the long side of the rectangle) because this was the closest situation to a 1D model and simulation. Indeed, it was our sample with the largest aspect ratio, and considering the front position at the centre of the long side, it could be assumed that the influence of the fronts in the perpendicular direction ( $\mathrm{x}$ fronts) was as little as possible, at least at the beginning. The distance of interest, noted y (see Fig. 1d), was the one measured and compared to the calculations. There was a certain interfacial width between the fluid and the packed regions. Measurements were performed from the centre of the interface. More details about the determination of the compaction front position by ImageJ are given in the Electronic Supplementary Information (Fig. S1). 
No water front could be detected in our experiments, thus justifying the use of an infinite capillary pressure in the calculations (see below). Actually, a water front necessarily occurs but, in our drying conditions and with our relatively small samples, it started after the compaction front had reached the centre of the sample. The kinetics of the water front could not be studied because it corresponded to the apparition of cracks.

In order to check for reproducibility of the compaction front positions versus time, the same experiment was repeated 4 times in the same conditions. First, we wanted to show (Fig. 2a), at least once, the direct outcome of an experiment: front position versus time in linear scales and real dimensions (length in centimetres, time in seconds). As can be seen, the front progressively accelerates, because the particle volume fraction increases in the fluid and the capillary suction of the wet gel zone becomes stronger as it length develops. Then, Fig. $2 b$ shows four successive results, an average of the four measurements and the corresponding standard deviation. Errors are due to slight variations in temperature, humidity and contact angle of the deposit on the substrate and an error in the front position measurement. The corresponding error bar was extended to all following measurements.
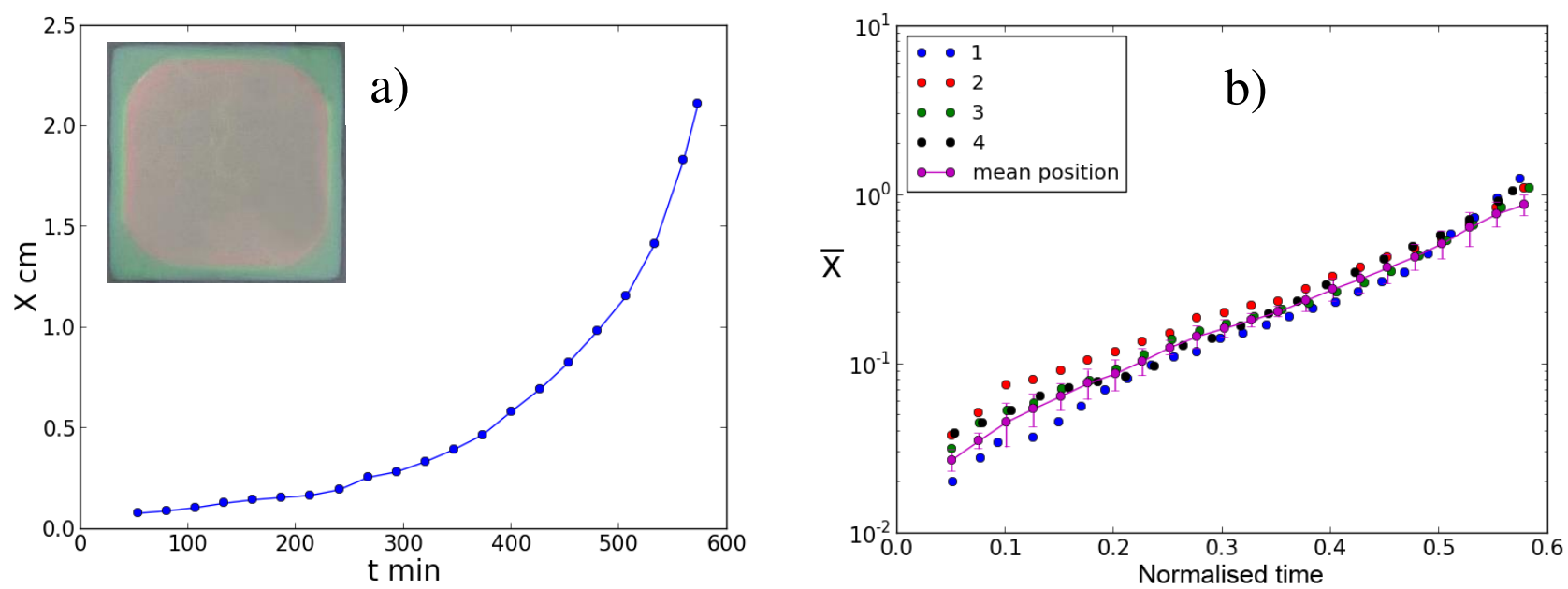

Fig. 2 Measured compaction front position versus time for a silica suspension (particle diameter 270 $\mathrm{nm}$; initial volume fraction 0.3$)$ dried on a glass substrate under controlled conditions $\left(23 \pm 2^{\circ} \mathrm{C}, 75 \pm 2 \%\right.$ $\mathrm{RH}$, still air). Initial deposit dimensions: $53 \mathrm{~mm} \times 53 \mathrm{~mm} \times 700 \mu \mathrm{m}$. a) Real time and length. b) Reproducibility check of front position, four repetitions. Time scaled on the characteristic time $\frac{H_{0}}{\dot{E}} \cdot \bar{X}$ the lateral distance scaled on the capillary length $\left(H_{0}\left(\frac{\gamma}{3 \eta_{0} \dot{E}}\right)^{1 / 4}\right.$ ) (values for $H_{0}, \dot{E}, \gamma, \eta_{0}$ indicated in the Experimental part). Insert: photography of the sample. 
Atomic Force Microscopy was used to visualize the morphology of the top surface of the dried films. A multimode AFM with a Nanoscope IV controller from Veeco (Digital Instrument, Santa Barbara, CA, USA) was used. The measurements were performed in tapping mode with a silicon tip with a maximal radius of curvature of $10 \mathrm{~nm}$. An image is shown in Fig. 1. From this kind of images, the maximum particle volume fraction could be estimated at $0.70 \pm 0.02$.

\section{Profilometry}

Film profiles were determined using an optical deflectometry method (Holomap ${ }^{\circledR}$ ) developed by Holo3, a small company specialized in optical measurements. ${ }^{39}$ A light source projects the image of a grid onto the sample. A CCD camera captures images of this grid reflected by the surface of the suspension. Both light source and camera are placed above the sample. The non-planar surface of the drying film creates a distortion of the image of the grid. An image processing software transforms the deformed grid into quantified information of the local slopes of the surface as well as XYZ coordinates to reconstruct the topography. The measured surface size was $25 \mathrm{~mm}$ x $25 \mathrm{~mm}$ with a vertical resolution of a few micrometres. A sketch of the setup is available is the Supplementary Information.

\section{Results and discussion}

\section{Evaporation at infinite $\mathrm{Lg}$}

We first report the results of our model in the limit Lcap $<<\operatorname{Lg}$, that is when the hydrostatic pressure plays a negligible role. This corresponds to the hypothesis of Routh and Russel model of lateral drying. ${ }^{9}$ Our simulation was run within the same set of assumptions and conditions as in the RR model: a deposit much longer than thick with a circular arc shape at the edge over a length of one reduced unit along $\mathrm{x}$, edge pinning throughout the drying process, constant and uniform evaporation rate all over the film, hard sphere monodisperse particles, initial and final particle volume fractions of 0.4 and 0.64 (random close packing), respectively; a homogeneous particle volume fraction and pressure along the film thickness, an infinite reduced capillary pressure (no water front), lateral convection only (no diffusion). Fig. 3 shows the time evolution of the film profile (a) and of the particle volume fraction (b) along the lateral direction calculated by our simulation tool. 

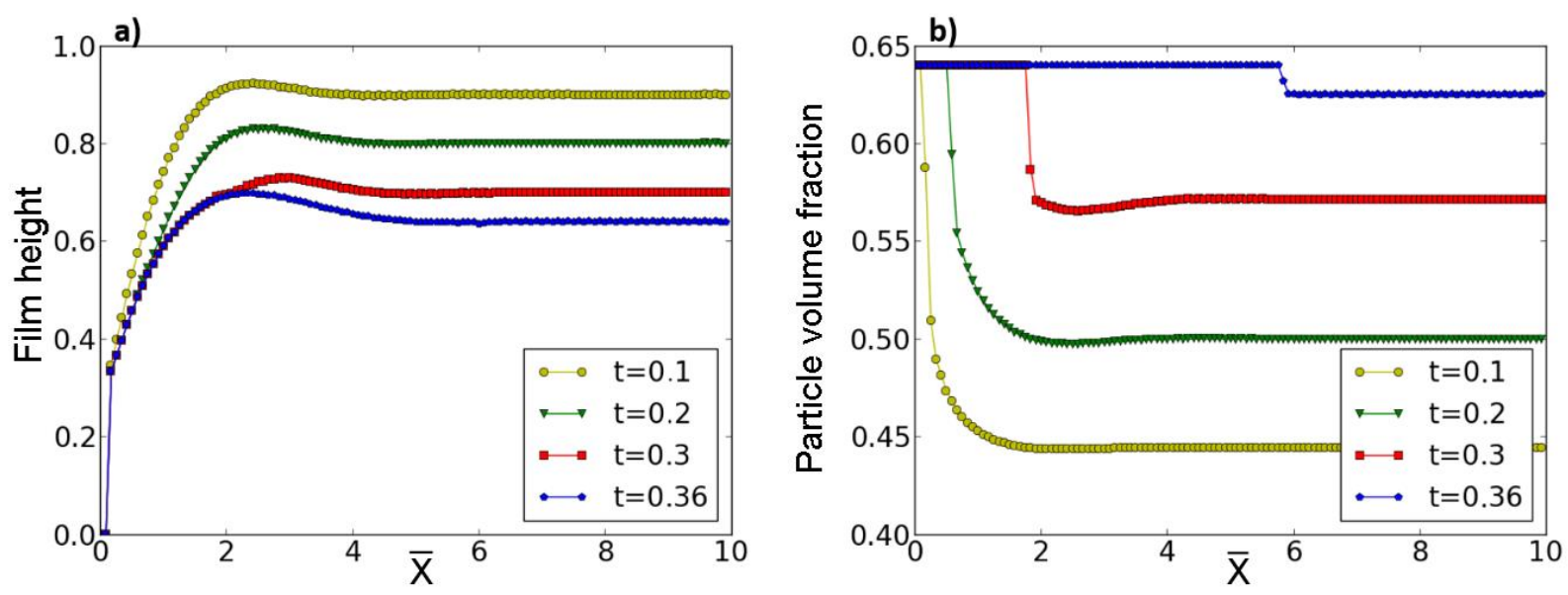

Fig. 3 Simulated film profile (a) and particle volume fraction (b) versus lateral dimension at different times during drying calculated using our cellular simulation in the conditions of the RR model. ${ }^{9}$ Film height scaled on the initial film thickness, lateral dimension $(\overline{\mathrm{X}})$ scaled on the capillary length $L_{c a p}$, time scaled on the characteristic time $\frac{H_{0}}{\dot{E}}$. Initial particle volume fraction $=0.4$. Volume fraction at close packing $=0.64$.

Our simulation results are identical to the ones calculated by Routh and Russel (see Fig. 5 in Ref. 9). The profile presents a bulge near the edge and then flattens out toward the centre. Far from the edge, the film height decreases with time due to vertical drying only. Depending on the conditions, especially initial and final particle volume fractions, a higher bulge and fast damping oscillations can be seen after the first maximum. This is shown in Fig. S2 in the supplementary information section for $\phi_{0}=0.3$ (initial volume fraction) and $\phi_{\max }=0.7$ (volume fraction at close packing). In Fig. 3b, it is shown that the wet gel / fluid zone interface (compaction front) is rather sharp and followed by a shallow depletion on the right. This depletion is more pronounced in the conditions of Fig. S2. The sharp compaction front can be attributed to the absence of diffusion. The depletion is linked to the film height profile and to the assumption of a constant evaporation rate over the whole film. The loss of a given amount of water per time unit will concentrate the dispersion less in a zone of higher height than in a neighbouring thinner zone. And, indeed, depletions are located in places corresponding to maxima in the film height profile (compare Fig. 3 a and b)(again, this is clearer in Fig. S2).

When the reduced capillary pressure is not infinite, a second front (water front) follows the compaction front at a certain distance, closer and closer when pressure reduces (not shown here, see Ref. 9 and 27).

There are several attempts in the literature to confront the RR model predictions and experiments. In the original theoretical paper itself, ${ }^{9}$ an interesting experiment was conducted 
consisting in drying a colloid under a mask with holes (perhaps the seminal work for evaporative lithography ${ }^{18}$ ). Faster evaporation under the holes led to particle convection towards these zones and bumps in the final deposit whereas covered areas were thinner, in qualitative agreement with the model. A couple of years later, Routh et al. ${ }^{40}$ took into account the deformation of soft particles in the wet gel and claimed "a semi-quantitative agreement" with experimental data. A thorough study in Keddie's team (Salamanca et $a l .^{26}$ ) used magnetic resonance profiling (MRP), also called magnetic resonance microscopy, to provide quantitative water profiles inside drying colloidal films in the vertical and lateral directions. The principal focus of the work was on the role of reduced capillary pressure in the appearance of the water front and its receding into the film. The model predictions about the main parameters controlling this reduced pressure (film thickness, evaporation rate, and particle size) were nicely confirmed. More recently, velocities of drying fronts were measured and compared to the RR calculation. ${ }^{27}$ Although general shapes and behaviours roughly agreed, fits of experimental curves by the model could not be considered good. It must be noted that the prediction of a bulge followed by oscillations in the profile has strong consequences as far as flow pattern inside the film is concerned. Nevertheless, this bulge has never been experimentally observed. We thus wish to consider the influence of hydrostatic pressure on the shape of the film surface.

\section{Contribution of hydrostatic pressure}

As shown above in the theoretical section, hydrostatic pressure in the film can be taken into account, essentially through the introduction of one more characteristic length, the capillary length in the classical sense of the dimension for which capillary and hydrostatic pressures correspond. In order to avoid confusion with the capillary length in the sense of Routh and Russel (written $L_{c a p}$ ), it appeared as $L_{g}$ (g stands for gravity). Actually, it is the $L_{c a p} / L_{g}$ ratio which is pertinent. From now on, initial and final particle volume fractions will be taken as 0.3 and 0.7 , respectively, because these values will allow comparisons with experimental data. The reduced capillary pressure in the wet gel is still considered as infinite (for calculation a value of 1000 was taken). Introduction of hydrostatic pressure besides Laplace pressure modifies the pressure field in the film and therefore the velocity field of the fluid, the film profile, the particle concentration profile and the compaction front velocity. 


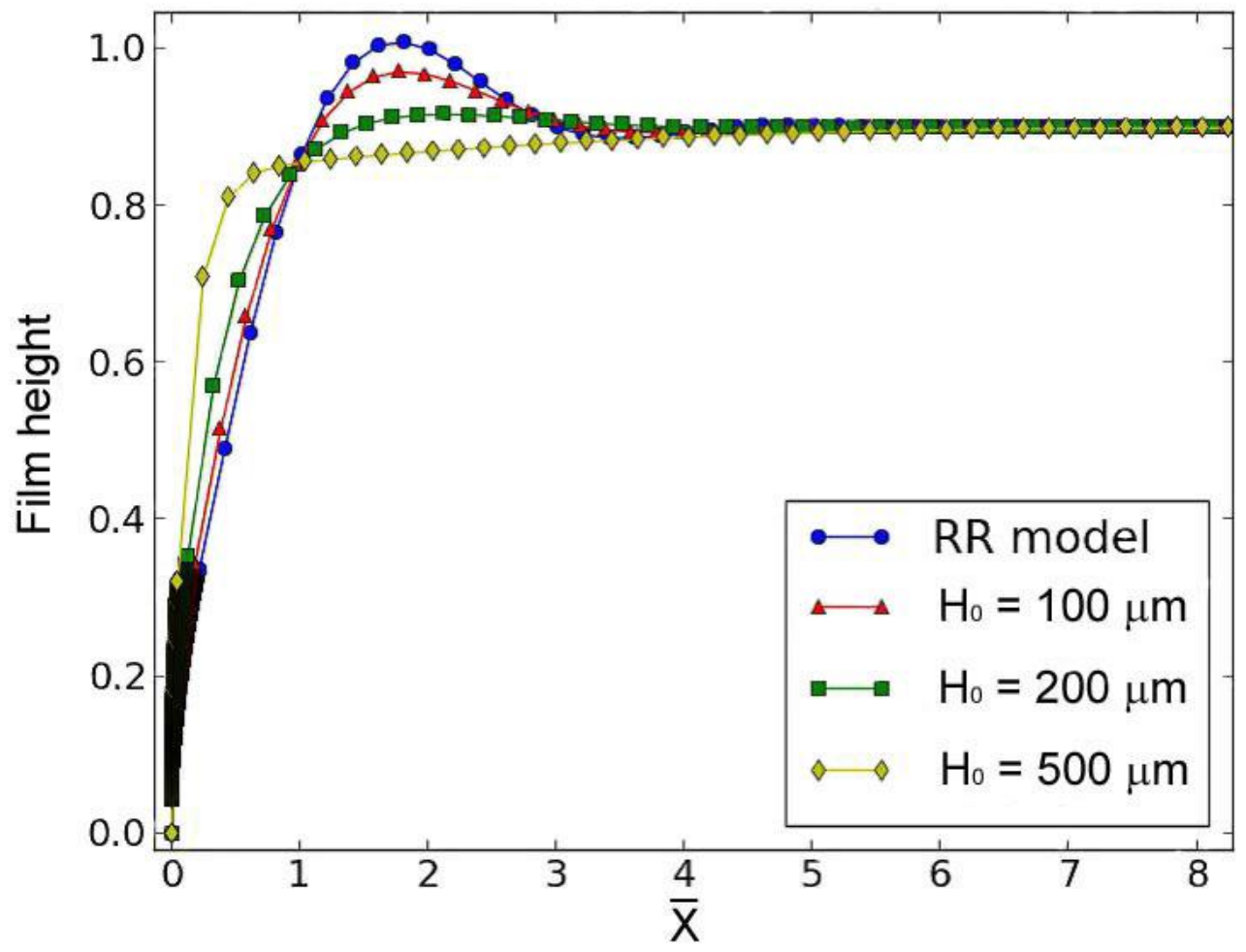

Fig. 4 Simulated film profile versus lateral dimension at time $=0.1$ for different initial thicknesses (i.e. different $L_{c a p} / L_{g}$ ratios) when taking hydrostatics into account. $L_{c a p} / L_{g}=1.5 ; 3 ; 7.5$ for $H_{0}=100 \mu \mathrm{m}$; $200 \mu \mathrm{m} ; 500 \mu \mathrm{m}$, respectively. $\gamma=70 \mathrm{~mJ} / \mathrm{m}^{2}, \eta_{0}=1$ Pa.s, $\dot{E}=10^{-8} \mathrm{~m} / \mathrm{s}, \rho=1000 \mathrm{~kg} / \mathrm{m}^{3}, \mathrm{~g}=9.81 \mathrm{~m} / \mathrm{s}^{2}$. Initial particle volume fraction $=0.3$. Volume fraction at close packing $=0.7$. Reduced capillary pressure $=1000$. Film height scaled on the initial film thickness, lateral dimension $(\overline{\mathrm{X}})$ scaled on $L_{\text {cap }}$, time scaled on the characteristic time $\frac{H_{0}}{\dot{E}}$.

Fig. 4 shows the film profile versus lateral dimension at time $=0.1$ for different initial thicknesses (i.e. different $L_{c a p} / L_{g}$ ratios) when taking hydrostatics into account. As expected, hydrostatics becomes more and more influent as the film thickness increases, progressively flattening the bulge near the edge and tending toward an equilibrium shape (Laplace pressure equilibrated by hydrostatic pressure). Note that, in Fig. 4, height and lateral dimension are scaled on different values depending on the initial film thickness, this might be misleading. Fig. $\mathrm{S} 3$ in supplementary information shows the same data in real dimension (meter). Fig. 5 shows the velocity of the fluid (particle plus water) in the fluid domain in the lateral direction, at times 0.2 and 0.3 , the contributions of Laplace pressure and hydrostatics appearing separately, and then the sum of the two. Laplace and hydrostatic pressure have opposite effects: Laplace pressure induces fluid flow from the edge towards the centre of the film whereas hydrostatic pressure induces flow towards the edge. The Laplace contribution changes shape between times 
0.2 and 0.3 , from a positive, continuously decreasing velocity to a weak, fast vanishing maximum. At around five capillary lengths from the edge, the fluid remains still.

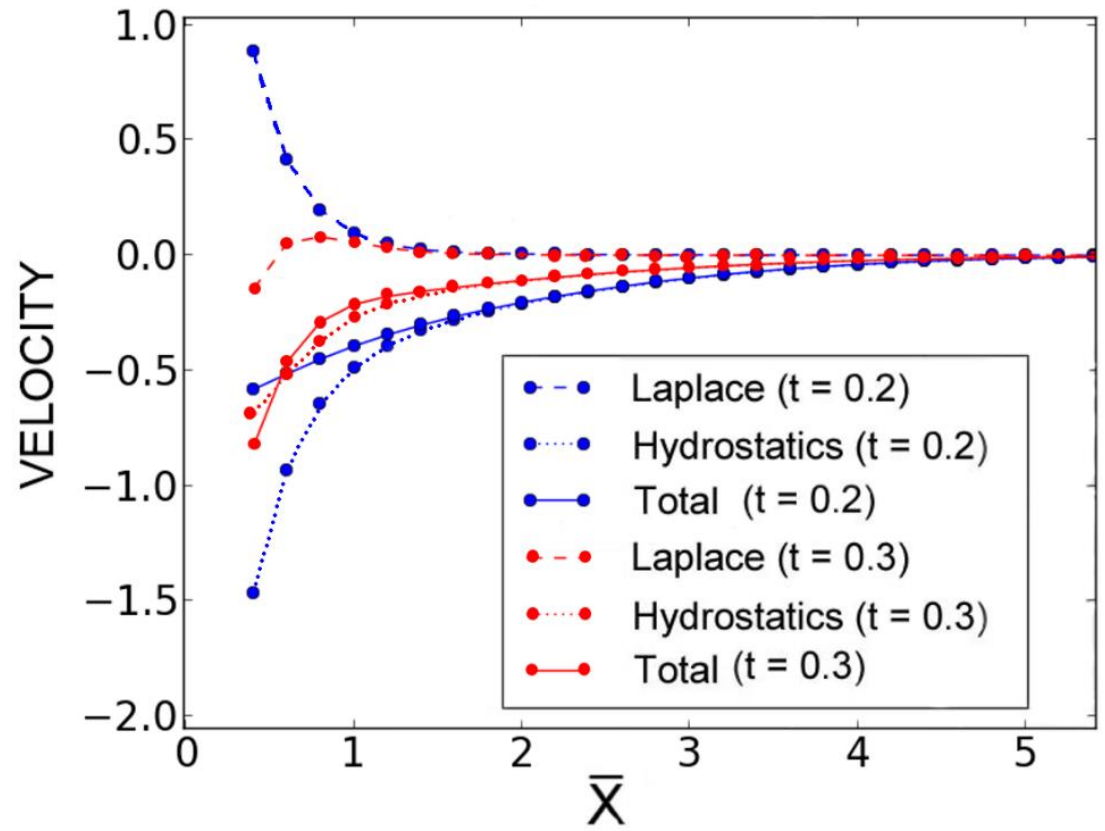

Fig. 5 Calculated velocity of fluid vs lateral dimension in a drying film showing the contributions of Laplace and hydrostatic pressures and the sum of the two at $\mathrm{t}=0.2$ and 0.3. $H_{0}=300 \mu \mathrm{m}\left(L_{c a p} / L_{g}=\right.$ 4.5). Velocities scaled on $\frac{L_{c a p} \dot{E}}{H_{0}}$, lateral dimension $(\overline{\mathrm{X}})$ scaled on $L_{\text {cap }}$, time scaled on $\frac{H_{0}}{\dot{E}}$.

In sharp contrast to the result shown in Fig. 5, i.e. a net flow of liquid extending to 5 capillary lengths ahead from the edge ("a far field flow"), a thorough SAXS study by Li et al. ${ }^{41}$ concluded that the number of particles at any given spot in the liquid film was conserved throughout the drying process and that there was no far field flow in a thin film of a silica colloid with $16 \mathrm{~nm}$ particles (diameter). The film, obtained by dip coating, was initially $10 \mu \mathrm{m}$ thick $(2 \mu \mathrm{m}$ after drying) with initial and final volume fractions of 0.14 and 0.68 , respectively. An important consequence of the absence of flow was that the dry film was homogeneous in thickness and microstructure. The authors asked themselves whether it would be possible to predict which deposits would present a far field flow like in a coffee drop and which ones would avoid macroscopic transport. The answer might be in the capillary length $L_{c a p}$. The data in Ref. 41 allow one to calculate $L_{c a p}=1 \mathrm{~mm}$ (in contrast to $L_{c a p}=3 \mathrm{~cm}$ in Fig. 5). The absence of detectable flow might be associated to this small value of $L_{c a p}$. The threshold $L_{c a p}$ value would remain to be determined. This would require a high sensitivity experimental technique to assess the presence or absence of particle flow. Li et al. ${ }^{41}$ also evidenced the presence of a transition region between the wet gel and the liquid far away from the compaction front where the particle 
concentration was higher than in the liquid. Interestingly, the width of this transition zone was in the order of $1 \mathrm{~mm}$, corresponding to $L_{c a p}$.

For a higher thickness $(700 \mu \mathrm{m})$, time evolution of film profile and particle concentration in the lateral direction are presented in Fig. 5. At this thickness, the bulge near the edge totally disappears (see Fig. 4) at all times. The profile looks exponential, in the form: $1-e^{-(x / a)}$ (a being a characteristics length specified later on). Interestingly, toward the end of the considered drying step $(\mathrm{t}=0.55)$, the edge presents a bump often seen indeed in dry films. The particle concentration profile is even sharper than in Fig. 3 with no depletion slightly away from the front. The absence of depletion is related to the fact that there is no longer a bulge in the fluid.
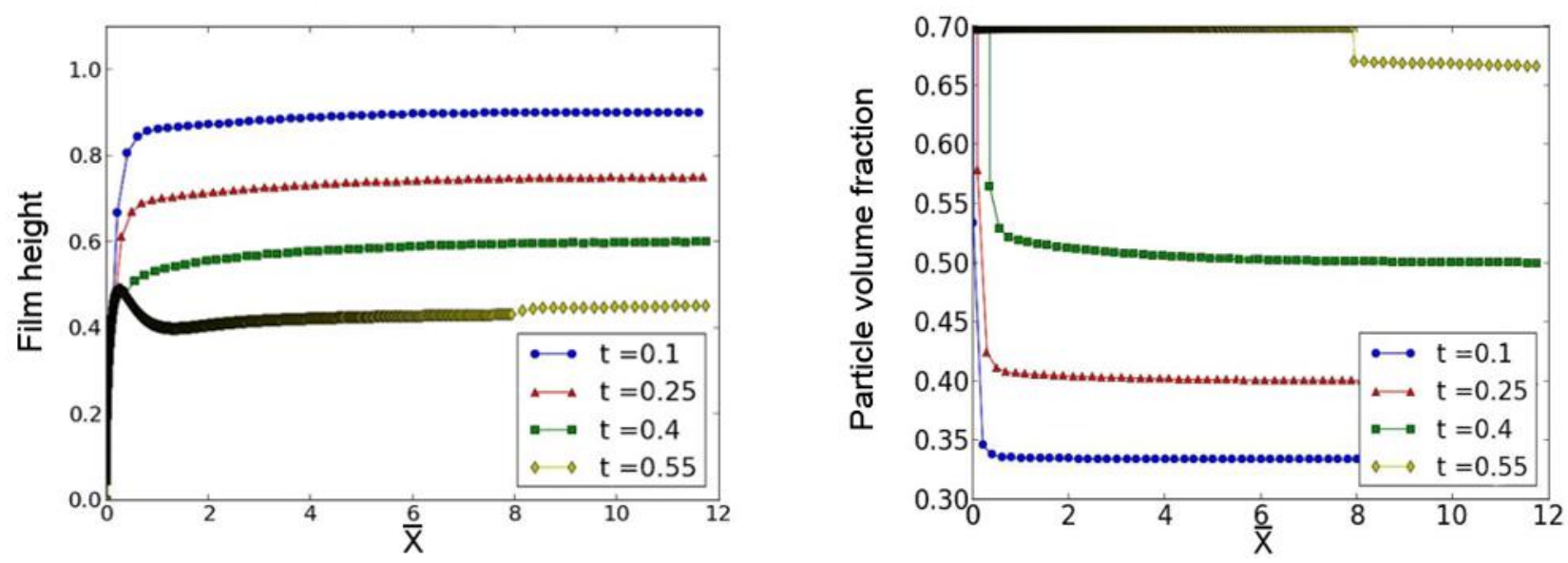

Fig. 6 Simulated film profile (left) and particle volume fraction (right) versus lateral dimension at different times. $H_{0}=700 \mu \mathrm{m}\left(L_{c a p} / L_{g}=10\right)$. Other parameters like in Fig. 4. Film height scaled on the initial film thickness, lateral dimension $(\overline{\mathrm{X}})$ scaled on $L_{\text {cap }}$, time scaled on $\frac{H_{0}}{\dot{E}}$.

With hydrostatics, the front position versus time keeps the same shape as in the RR model (Fig. S4 and Fig. 5 in Ref. 9) but more and more shifted toward higher times as thickness increases. Scaling and semi log plot make this S4 figure misleading (contrary to what the curves suggest, in reality, the front velocity increases monotonically ${ }^{27}$ ) and it will not be commented further. All we wanted to mention was the similarity of the shapes.

\section{Introduction of cooperative diffusion}

The Routh and Russel model does not include diffusion in the horizontal direction, only convection. In terms of Peclet number (Pe, ratio of convection over diffusion), the RR model comes down to an infinite horizontal Pe. This is possibly an excessive approximation. 
In the case of a dispersion with repulsive particles concentrating by drying, it is not the diffusion coefficient of an individual particle which is relevant but a cooperative diffusion coefficient describing the collective motion of an ensemble of particles. Actually, there are three diffusion coefficients of interest. The well-known Stokes Einstein diffusion coefficient (often written $\mathrm{D}_{0}$ ) describes the Brownian motion of a single particle isolated in a highly diluted dispersion. In a more concentrated dispersion, the motion of an individual particle is influenced by the presence of its neighbours. It is described by what is called the self diffusion coefficient $\left(D_{S}\right)$. Finally, there is the cooperative diffusion coefficient $\left(D_{\text {coop }}\right)$, the one of most interest to us, which describes the relaxation of a particle concentration gradient through a collective process. ${ }^{42} \mathrm{D}_{\text {coop }}$ is the coefficient appearing in Fick's first diffusion law. Upon concentration increase, the self diffusion coefficient decreases because of the motion hindrance by the neighbouring particles (cage effect) whereas the cooperative diffusion coefficient increases, accelerating the equilibration of concentration distributions via cooperative motion. ${ }^{34}$ Based on references 34 and 42, a quantitative expression for $\mathrm{D}_{\text {coop }}$ was implemented in our simulation, ${ }^{29}$ showing that, when electrostatic forces are taken into account, the cooperative diffusion coefficient sharply increases as the volume fraction reaches a certain threshold. These forces are under the dependence of the salt concentration in the dispersion, effect taken into account through the Debye length.

Introduction of horizontal diffusion indeed makes the compaction front less sharp than in Fig. 6 (not shown) and slows down the inward motion of the front (Fig. 7). Fig. 7 shows the front position versus time calculated following the RR model, then in the case of the introduction of the hydrostatic pressure and finally with diffusion (i.e. hydrostatics plus diffusion). Increasing the Debye length from 2 to $5 \mathrm{~nm}$, further delays the start of the front and decreases its velocity through an increase in the dimensionless cooperative diffusion coefficient $(\bar{D})$.

Data in Fig. 7 correspond to an initial film thickness of $300 \mu \mathrm{m}$. Increasing the film thickness enhances the contrast between the RR model and ours including hydrostatics (Fig. S4) and the dimensionless cooperative diffusion coefficient becomes weaker. As a consequence, the green, red and yellow curves in Fig. 7 tend to merge. 


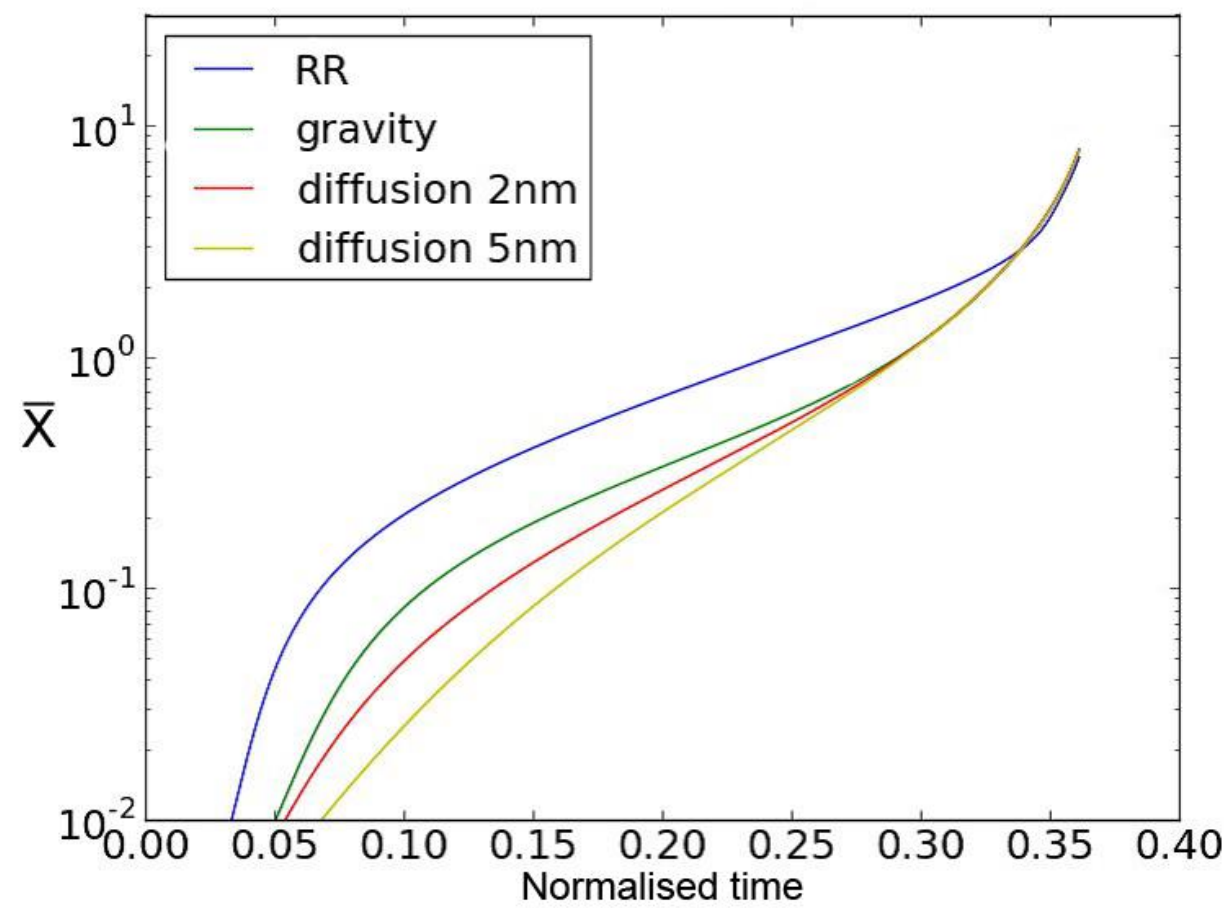

Fig. 7 Simulation of compaction front position versus time according to RR (blue), taking hydrodynamics into account (green) and adding diffusion (red and yellow) with two Debye lengths (2 and $5 \mathrm{~nm}$ ), as indicated. Lateral dimension $(\overline{\mathrm{X}})$ scaled on $L_{\text {cap }}$, time scaled on $\frac{H_{0}}{\dot{E}}$. $H_{0}=300 \mu \mathrm{m}$. Other parameters like in Fig. 4.

Peclet numbers in drying models are often calculated with parameters corresponding to the initial state of the film and then considered constant. Here, we speak of the horizontal Peclet number. It was defined as: $\frac{u_{x} L_{c a p}}{D_{c o o p}}$ with $u_{x}$ the fluid velocity in the lateral direction, $L_{c a p}$ the RR capillary length, and $D_{\text {coop }}$ calculated like in Ref. 29. From our simulation, we can extract values allowing us to calculate $\mathrm{Pe}$ as a function of time and position. It is interesting to show how $\mathrm{Pe}$ varies upon drying (Fig. 8). The peak near the compaction front (at around one reduced lateral length unit, i.e. 1 Lcap) is due to the high convective velocity at this place. Further away from the front, velocity sharply decreases. Closer to the front, Pe goes down because of a strong diffusion coefficient related to a high particle concentration in the immediate vicinity of the front. As drying proceeds, Pe decreases. This is due to the increase of $D_{\text {coop }}$ linked to the gradual increase of the particle concentration. 


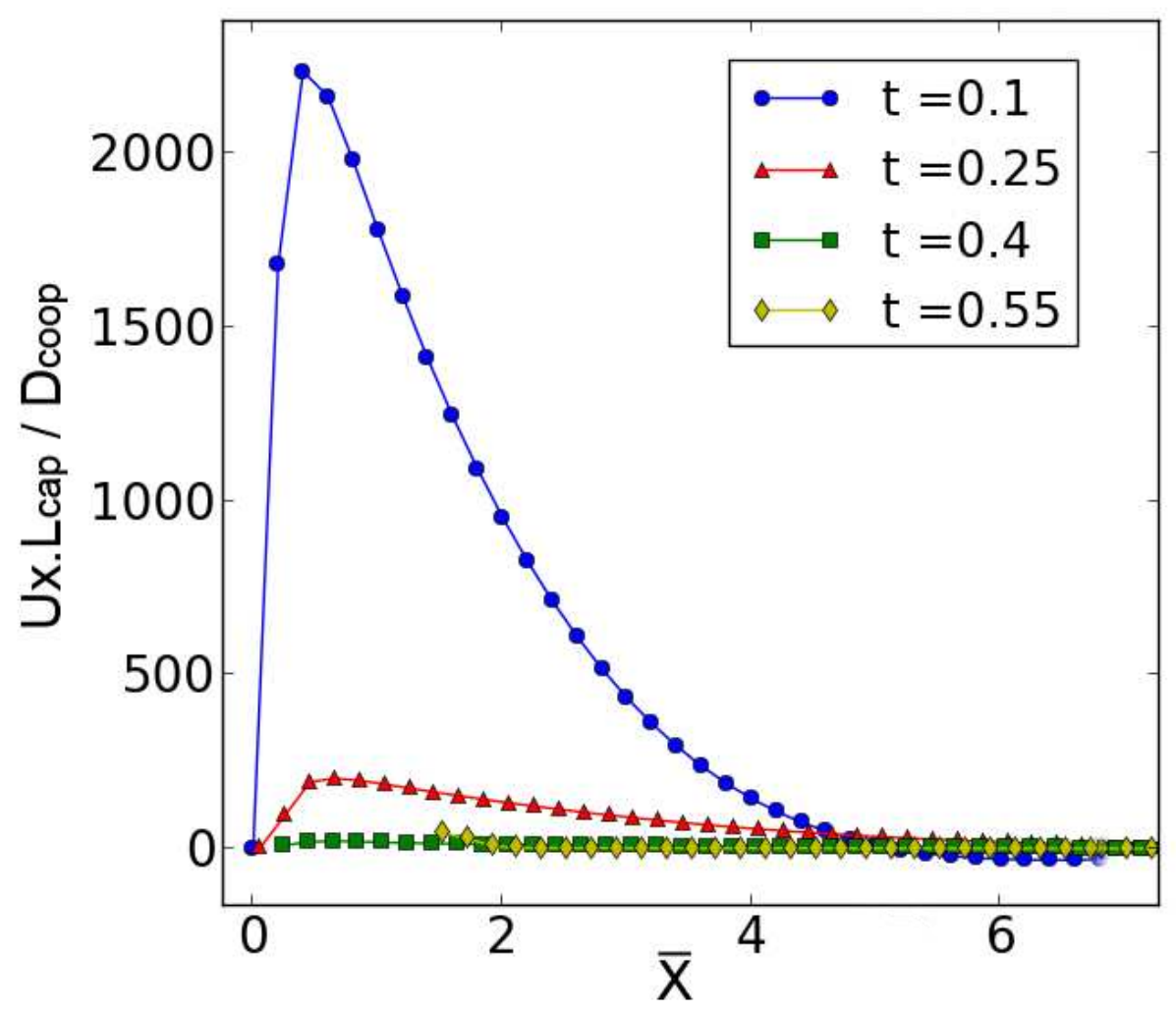

Fig. 8 Calculated horizontal Peclet number versus lateral dimension. Lateral dimension $(\overline{\mathrm{X}})$ scaled on $L_{\text {cap }}$, time scaled on $\frac{H_{0}}{\dot{E}}$. $H_{0}=300 \mu \mathrm{m}$. Debye length $=5 \mathrm{~nm}$. Particle diameter $=270 \mathrm{~nm}$. Other parameters like in Fig. 4.

\section{Comparisons with experiments}

In the RR model, the shape of the profile is a critical characteristic of the film in the liquid state. In particular, there is a bulge close to the edge at early drying stages, or at the solid / liquid interface later on (Fig. 3 and Fig. 4). This bulge tends to disappear when the hydrostatic pressure is taken into account in the simulation, this behaviour being more and more marked when film thickness increases (Fig. 4 and Fig. 6). In order to check this aspect experimentally, the film profile was determined using the optical technique described in the experimental section (Fig. 9). 

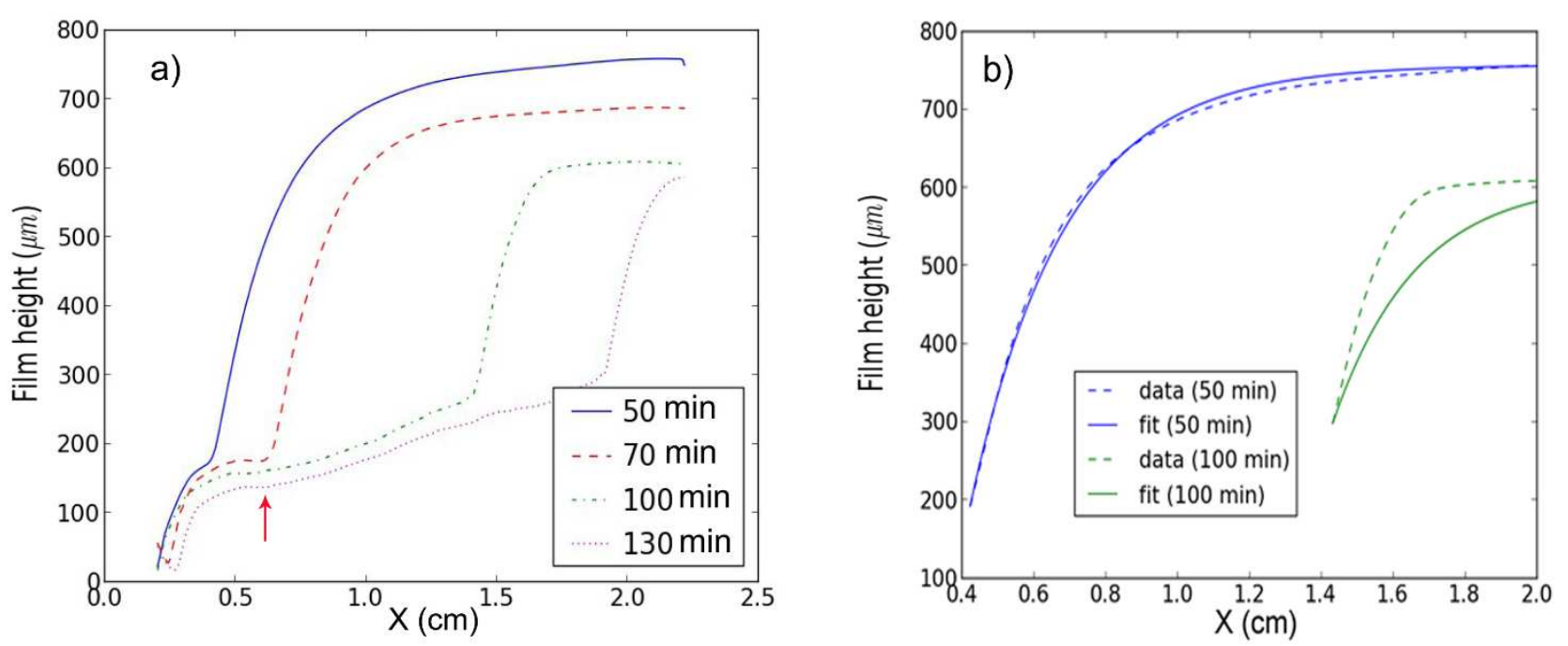

Fig. 9 Experimental film height versus lateral dimension at different times. Silica particles (diameter 270 $\mathrm{nm}$ ). Initial volume fraction 0.3. $H_{0}=700 \mu \mathrm{m}$. Circular deposit of diameter $6 \mathrm{~cm}$. Standard drying conditions. a) Experiments. The red arrow shows the compaction front position at $70 \mathrm{~min}$, as an example. b) Exponential fits.

The acquisition zone of the camera being limited to a surface of $(2.5 \times 2.5) \mathrm{cm}$, only half the deposit is shown in Fig. 9a. (obviously, the centre is at the right side of the figure). It is immediately evident that no bulge exits in the profile of the fluid zone. The interface between the wet gel and the liquid dispersion (compaction front) is also clear in the figure (for instance, red arrow at $70 \mathrm{~min}$ ), as well as the motion of the compaction front to the right as drying proceeds. The height of the film far from the edge decreases with time due to vertical drying. The shape at early times suggests an equilibrium profile (hydrostatic pressure equilibrating Laplace pressure). This would imply a fit by a function:

$h(x)=H_{\max }\left(1-e^{-\left(x / L_{g}\right)}\right)$ with $H_{\max }$ the maximum height far from the edge and $L_{g}=$ $\left(\frac{\gamma}{\rho g}\right)^{1 / 2}$ the capillary length in the usual sense. Fig. $9 \mathrm{~b}$ shows that, at 50 min, indeed, the fit is very good (standard deviation error $4.7 \times 10^{-6}$ ) with $L_{g}=2.65 \mathrm{~mm}$, the expected value for water. Whereas, at $100 \mathrm{~min}$, the fit strongly deteriorates (even when the increased density of the dispersion is taken into account) indicating an increased contribution of Laplace pressure. These profiles are very close to the ones in Fig. 6 (left) (except for the shape of the wet gel). This is a strong indication in favour of the introduction of hydrostatics in the model.

Next, the simulation was checked via the front position versus time with the sample of rectangular shape and dimensions $120 \mathrm{~mm}$ x $20 \mathrm{~mm}$. The simulation / experiment comparison 
is shown in Fig. 10. Experimental data start at time 0.15. At shorter times, when the front was still close to the edge, it was not clear enough to enable us to measure its position. The agreement of experimental data with the simulated curve taking into account the contribution of hydrostatics is very good, except for the deviation at the end. The fact that the real sample reaches the final drying state considered here (close packed particles over the whole sample) before the simulated one and the disagreement at the end can be attributed to the contributions of the two $\mathrm{x}$ fronts and to the $\mathrm{y}$ front on the opposite side of the rectangle getting close.

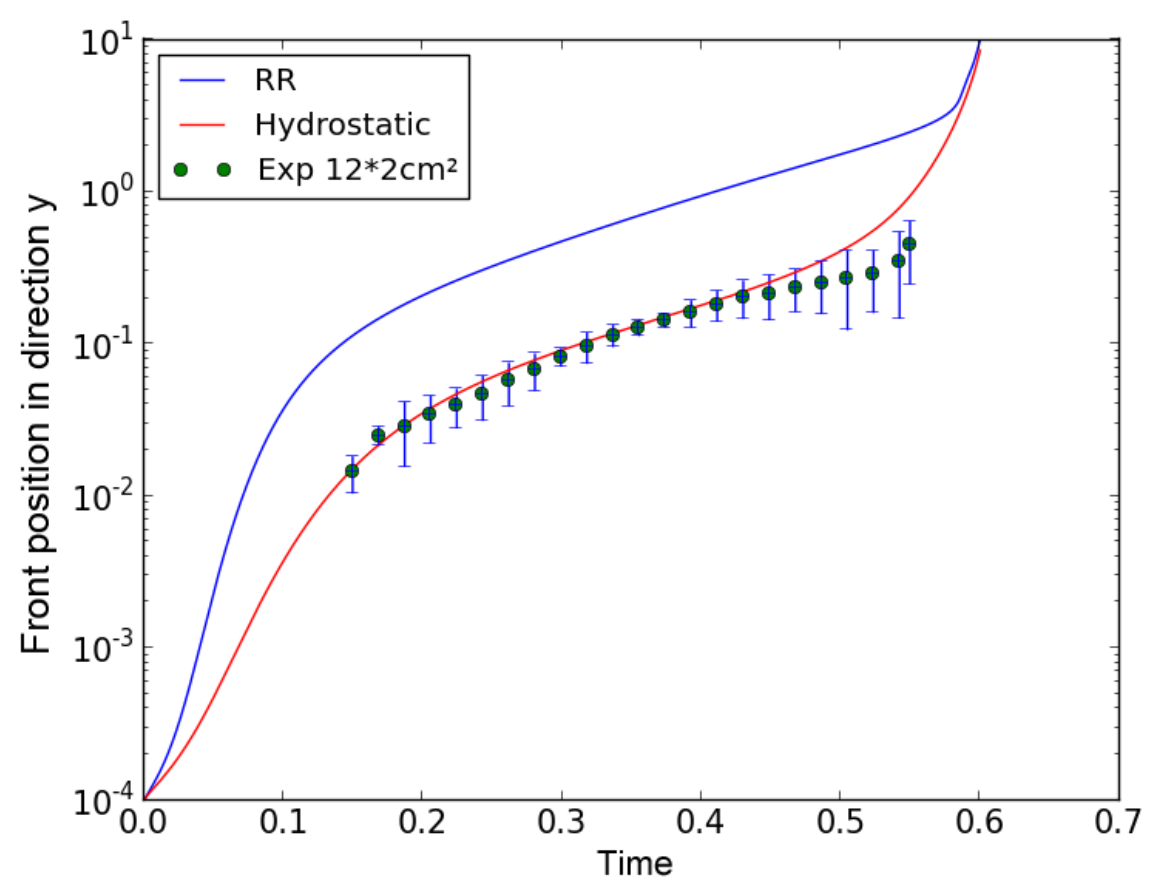

Fig. 10 y front position versus time for a rectangular sample $120 \mathrm{~mm} \times 20 \mathrm{~mm} \times 700 \mu \mathrm{m}$ dried in standard controlled conditions $\left(23 \pm 2^{\circ} \mathrm{C}, 75 \pm 2 \% \mathrm{RH}\right.$, still air). Comparison with the Routh and Russel model (blue) and with our simulation integrating hydrostatics (red). Experimental points in green.

The contribution of diffusion could not be checked in our experimental conditions $\left(H_{0}=700\right.$ $\mu \mathrm{m})$ because, at this thickness, the curves with hydrostatics and diffusion are almost superposed. New sets of experiments should be designed for that purpose. 


\section{Conclusion}

Our simulation approach was able to reproduce the results of the RR model in a less conceptually and mathematically demanding way. Indeed, the use of Euler coordinates (our case), i.e. coordinates fixed in space, is easier to apprehend than Lagrange coordinates which follow the moving boundaries (in the RR model) leading to very complex calculations. Furthermore, progressive introduction of improvements in the physical laws accounted for in the simulation was relatively easy. Taking in consideration the hydrostatic pressure in addition to Laplace pressure modifies the film profile, the velocity field in the fluid and slows down the propagation of the compaction front. When horizontal diffusion is introduced through the cooperative diffusion coefficient which depends on the ionic strength of the medium, the front is further delayed. Calculations showed that the effect of diffusion on the compaction front velocity was only significant for the smallest film thicknesses. When confronted to experiment, the simulation was surprisingly well validated: film profiles (in the fluid domain only) and front positions versus time corresponded quite well. It was surprising that a $1 \mathrm{D}$ simulation could so nicely fit experiments that are 3D in nature. To check the contribution of diffusion, our films were too thick to allow us to draw conclusions on this point.

Two side aspects of this study are (i) the highlighting that considering a Peclet number fixed by the initial conditions and constant throughout the drying process can lead to marked errors in models and simulations; and (ii) the suggestion that the capillary length $L_{c a p}$ could be the discriminating factor between films where far field flows take place, leading to heterogeneous dry deposits (high $L_{c a p}$ ), and others where particles do not move long distances, leading to homogeneous deposits (low $L_{c a p}$ ). The practical importance of this aspect is obvious. The controlling parameters would then simply be the initial film thickness, the surface tension, the viscosity of the dispersion and the global drying rate. Of course, this suggestion deserves thorough validation and an experimental determination of the threshold $L_{c a p}$ value.

Several ways of improvements of this study exist and can be mentioned. First, experiments with varying thicknesses should be performed. Then, the simulation does not at all account for the shape of the dry deposit. This is a major weakness that should be addressed with high priority. Also, deformable particles should be considered in the situation where drying goes further, until total elimination of water. This was already done in the vertical direction, ${ }^{29}$ the concepts and numerical tools are ready to be adapted and used. Similarly, of great interest would be to simulate the distribution of small molecules like surfactants in the horizontal direction, also already done in the vertical direction. ${ }^{30}$ 
In the meantime, our next contribution, ready to be published, will be on a 2D simulation in the plane of the film, able to account for the influence of the deposit shape on the front velocities in different directions.

\section{Conflicts of interest}

There are no conflicts of interest to declare.

\section{Acknowledgements}

We gratefully acknowledge Dr. L. Jacomine for helpful assistance in the profilometry measurements.

\section{References}

1. H.Y. Erbil, Control of stain geometry by drop evaporation of surfactant containing dispersions, Adv. Colloid. Interf. Sci. 2015, 222, 275-290.

2. W.B. Russel, Mechanics of Drying Colloidal Dispersions: Fluid/Solid Transitions, Skinning, Crystallization, Cracking, and Peeling, AIChE J. 2011, 57, 1378-1385.

3. A.F. Routh, Drying of thin colloidal films, Rep. Prog. Phys. 2013, 76, 046603.

4. S.G. Croll, Drying of latex paint, J. Coat. Technol. 1986, 58 (734), 41-49.

5. J.C.H. Hwa, Mechanism of film formation from latexes. Phenomenon of flocculation, $J$. Polym. Sci. A 1964, 2, 785-796.

6. M. Okubo, T. Takeya, Y. Tsutsumi, T. Kadooka, T. Matsumoto, Asymmetric porous emulsion films, J. Polym. Sci. Polym. Chem. Ed. 1981, 19, 1-8.

7. Y. Ma, H.T. Davis, L.E. Scriven. Microstructure development in drying latex coatings. Prog.Org. Coat. 2005, 52, 46-62.

8. A.F. Routh, W.B. Zimmerman, Distribution of particles during solvent evaporation from films, Chem. Eng. Sci. 2004, 59, 2961-2968.

9. A.F. Routh, W.B. Russel, Horizontal drying fronts during solvent evaporation from latex films, AICHE J. 1998, 44, 2088-2098. 
10. S.G. Yiantsios, B.G. Higgins, Marangoni flows during drying of colloidal films, Phys. Fluid. 2006, 18, 082103.

11. J. Feng, M.A. Winnik, R.R. Shivers, B. Clubb, Polymer blend latex films: morphology and transparency, Macromolecules 1995, 28, 7671- 7682.

12. T.A. Thorstenson, J.B. Huang, M.W. Urban, K. Haubennestel, Mobility and distribution of silicone additives in coatings: a spectroscopic study, Prog. Org. Coat. 1994, 24, 341-358.

13. A. Tzitzinou, P.M. Jenneson, A.S. Clough, J.L. Keddie, J.R. Lu, P. Zhdan, K.E. Treacher, R. Satguru, Surfactant concentration and morphology at the surface of acrylic latex films, Prog Org Coat. 1999, 35, 89-99.

14. M. Schulz, H. Römermann, K. Pohl, C. Chindawong, D. Johannsmann, Latex Films with In-Plane Composition Gradients Caused by Lateral Drying, Soft Mater. 2015, 13, 138-143.

15. R.E. Trueman, E.L. Domingues, S.N. Emmett, M.W. Murray, J.L. Keddie, A.F. Routh, Autostratification in Drying Colloidal Dispersions: Experimental Investigations. Langmuir $2012,28,3420-3428$.

16. A. Fortini, I. Martín-Fabiani, J.L. De La Haye, P.Y. Dugas, M. Lansalot, F. D’Agosto, E. Bourgeat-Lami, J.L. Keddie, R.P. Sear, Dynamic Stratification in Drying Films of Colloidal Mixtures, Phys. Rev. Lett. 2016, 116, 118301.

17. I. Nikiforow, J. Adams, A.M. König, A. Langhoff, K. Pohl, A. Turshatov, D. Johannsmann, Self-Stratification During Film Formation from Latex Blends Driven by Differences in Collective Diffusivity, Langmuir 2010, 26, 13162-13167.

18. D.J. Harris, H. Hu, J.C. Conrad, J.A. Lewis, Patterning Colloidal Films via Evaporative Lithography, Phys. Rev. Let. 2007, 98, 148301.

19. C. Parneix, P. Vandoolaeghe, V.S. Nikolayev, D. Quéré, J. Li, B. Cabane, Dips and Rims in Dried Colloidal Films, Phys. Rev. Let. 2010, 105, 266103.

20. A. Georgiadis, A.F. Routh, M.W. Murray, J.L. Keddie, Bespoke periodic topography in hard polymer films by infrared radiation-assisted evaporative lithography, Soft Matter 2011, 7, 11098-11102.

21. R.D. Deegan, O. Bakijin, T.F. Dupont, G. Huber, S.R. Nagel, T.A.Witten, Capillary flow as the cause of ring stains from dried liquid drops, Nature 1997, 389, 827-829. 
22. R.D. Deegan, O. Bakajin, T.F. Dupont, G. Huber, S.R. Nagel, T.A. Witten, Contact line deposits in an evaporating drop, Phys. Rev. E 2000, 62, 756-765.

23. H.M. van der Kooij, G.T. van de Kerkhof, J. Sprakel, A mechanistic view of drying suspension droplets, Soft Matter 2016, 12, 2858-2867.

24. J.L. Keddie, A.F. Routh, Fundamentals of Latex Film Formation: Processes and Properties, Springer: Dordrecht, The Netherlands, 2010.

25. M.A. Winnik, J. Feng, Latex blends: an approach to zero VOC coatings, J. Coat. Technol. 1996, 68 (852), 39-50.

26. J.M. Salamanca, E. Ciampi, D.A. Faux, P.M. Glover, P.J. McDonald, A.F. Routh, A.C.I.A. Peters, R. Satguru, J.L. Keddie, Lateral Drying in Thick Films of Waterborne Colloidal Particles, Langmuir 2001, 17, 3202-3207.

27. V. Divry, A. Gromer, M. Nassar, C. Lambour, D. Collin, Y. Holl, Drying Mechanisms in Plasticized Latex Films: Role of Horizontal Drying Fronts, J. Phys. Chem. B 2016, 120, $6791-6802$.

28. H.M. van der Kooij, G.T. van de Kerkhof, J. Sprakel, A mechanistic view of drying suspension droplets, Soft Matter 2016, 12, 2858-2867.

29. A. Gromer, M. Nassar, F. Thalmann, P. Hébraud, Y. Holl, Simulation of Latex Film Formation Using a Cell Model in Real Space: Vertical Drying, Langmuir 2015, 31, 10983-10994.

30. A. Gromer, F. Thalmann, P. Hébraud, Y. Holl, Simulation of Vertical Surfactant Distributions in Drying Latex Films, Langmuir 2017, 33, 561-572.

31. B. Chopard, M. Droz, Cellular Automata Modeling of Physical Systems. Cambridge University Press, 1998.

32. A. Wuerger, Thermal non-equilibrium transport in colloids, Rep. Prog. Phys. 2010, 73, 126601.

33. P. G. de Gennes, F. Brochard-Wyart, D. Quéré, Capillarity and Wetting Phenomena. Drops, Bubbles, Pearls, Waves. Springer-Verlag New York, 2004.

34. A.M. König, T.G. Weerakkody, J.L. Keddie, D. Johannsmann, Heterogeneous Drying of Colloidal Polymer Films: Dependence on Added Salt, Langmuir 2008, 24, 7580-7589.

35. G. Taylor, Dispersion of soluble matter in solvent flowing slowly through a tube, Proc. $R$. Soc. A, 1953, 219(1137),186-203. 
36. R.K. Iler, The chemistry of silica, Wiley Interscience, 1979.

37. K. von der Ehe, D. Johannsmann, Maps of the stress distributions in drying latex films, Rev. Sci. Instrum. 2007, 78 (11), 113904.

38. V. Lazarus, L. Pauchard, From craquelures to spiral cracks patterns: influence of layer thickness on the crack patterns induced by desiccation, Soft Matter 2011, 7, 2552-2559.

39. J.P. Chambard, P. Danieau, Dispositif d'analyse et d'imagerie d'une surface par deflectométrie et procédé correspondant, French patent 2015, FR 3018603 A1.

40. A.F. Routh, M.S. El-Aasser, J. Tang, W.B. Russel, Process Model for Latex Film Formation: Optical Clarity Fronts, J. Coat. Technol. 2001, 73 (916), 41-48.

41. J. Li, B. Cabane, M. Sztucki, J. Gummel, L. Goehring, Drying Dip-Coated Colloidal Films, Langmuir 2012, 28, 200-208.

42. W.R. Bowen, A. Mongruel, Calculation of the collective diffusion coefficient of electrostatically stabilised colloidal particles, Colloid Surf. A. 1998, 138, 161-172. 
Horizontal drying fronts in films of colloidal dispersions: influence of hydrostatic pressure and collective diffusion

M. Nassar, A. Gromer, D. Favier, F. Thalmann, P. Hébraud, Y. Holl

Electronic Supplementary Information

Schemes of drying fronts

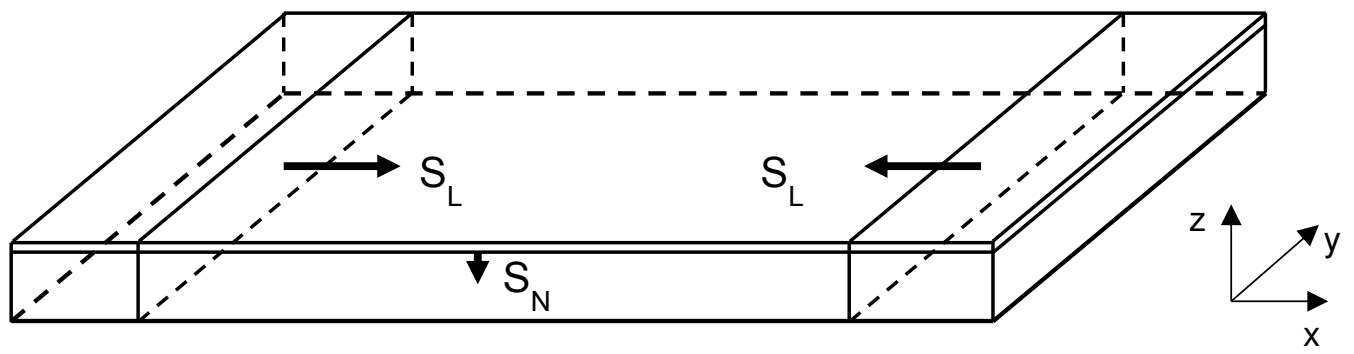

Schematic (above) of a drying colloid showing normal (vertical, $\mathrm{z}$ direction) and lateral (horizontal, $x$ direction) drying fronts moving at speeds $S_{N}$ and $S_{L}$, respectively. $S_{L}>S_{N}$. For clarity, lateral drying fronts in the y direction are not represented but also exist.

In the case of rigid particles, when one zooms onto a horizontal drying front (below) one sees that two fronts can be distinguished: a compaction front where particles agglomerate and a water front separating the wet and dry gel regions.

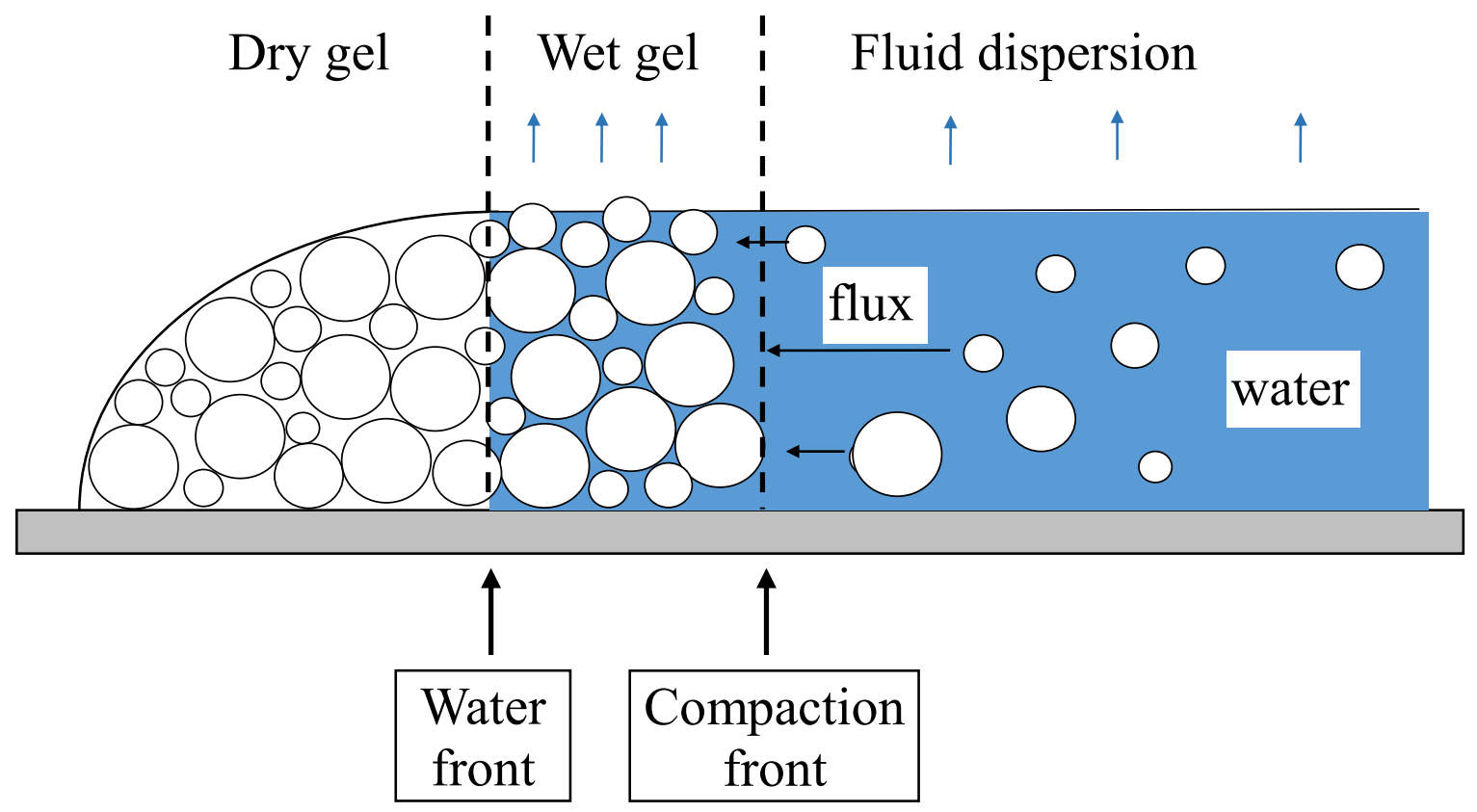


In the following, we derive the fluid velocity in the fluid domain within the lubrication approximation in presence of hydrostatic pressure, for a simple case (1D) of a film spreading on a substrate with $P_{A}, P_{B}, P_{C}, P_{D}$ the pressures at different points in the film:

$$
P_{A}-P_{B}+P_{B}-P_{C}+P_{C}-P_{D}+P_{D}-P_{A}=0
$$

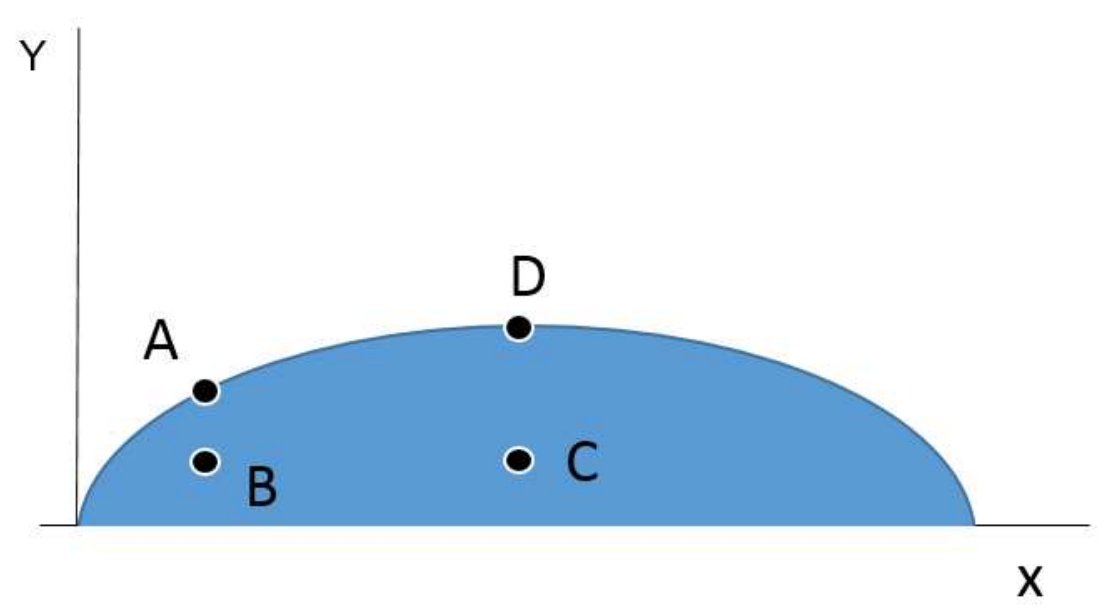

Schematic of a sample during drying. Each point on the schematic has a specific pressure.

Where:

- $P_{A}-P_{D} \sim 0$,

- $P_{A}-P_{B} \sim \gamma \frac{d^{2} h(x)}{d x^{2}}, \mathrm{~h}(\mathrm{x})=$ height of the film at position $\mathrm{x}$.

- $P_{B}-P_{C} \sim \int_{C}^{B} \vec{\nabla} p \cdot \overrightarrow{d l}=\int_{x_{C}}^{x_{B}} \frac{d p}{d x} \cdot d x$,

- $P_{C}-P_{D} \sim+\rho g\left(h_{\infty}-h_{C}\right)=\rho g\left(h_{\infty}-h_{B}\right)=\rho g\left(h_{\infty}-h\left(x_{B}\right)\right)$ here $h_{\infty}$ represents the maximum height of the film.

The characteristic time of establishment of hydrostatic pressures is $\sim H_{0} / C_{s}$. Where $H_{0}$ is the initial height of the sample and $C_{s}$ is a constant.

The integral of the pressure circuit gives:

$$
\gamma \frac{d^{2} h\left(x_{B}\right)}{d x^{2}}+\int_{x_{C}}^{x_{B}} \frac{d p}{d x} \cdot d x+\rho g\left(h_{\infty}-h\left(x_{B}\right)\right)=0
$$

Within the lubrication approximation, the profile of horizontal fluid velocity is given by Poiseuille's law. The mean velocity in the horizontal direction is

$$
<u>=-K \frac{d P}{d x}
$$

The characteristic time of Poiseuille is: 


$$
\frac{H_{0}^{2}}{\mu}=\frac{\rho H_{0}^{2}}{\eta}=\tau_{P}
$$

With $\mu$ the kinematic viscosity and $\eta$ the dynamic viscosity. It has to be noted that every relaxation faster than $\tau_{P}$ is likely not to be well described using the lubrication approximation.

$$
\begin{gathered}
K=\frac{h^{2}}{3 \eta} \\
\tau_{P}=3 \rho K=\frac{\rho h^{2}}{\eta}
\end{gathered}
$$

Using these equations in the pressure circuit gives:

$$
\begin{gathered}
\gamma \frac{d^{2} h\left(x_{B}\right)}{d x^{2}}+\int_{x_{C}}^{x_{B}}-\frac{<u>(x)}{K(x)} d x+\rho g\left(h_{\infty}-h\left(x_{B}\right)\right)=0 \\
\gamma \frac{d^{3} h\left(x_{B}\right)}{d x^{3}}-\frac{<u>\left(x_{B}\right)}{K\left(x_{B}\right)}-\rho g \frac{d h}{d x_{B}}=0
\end{gathered}
$$

Therefore:

$$
h<u>=K\left[\gamma \frac{d^{3} h}{d x^{3}}-\rho g \frac{d h}{d x}\right] h=\frac{h^{3}}{3 \eta}\left[\gamma \frac{d^{3} h}{d x^{3}}-\rho g \frac{d h}{d x}\right]
$$

The variation of $\mathrm{h}$ within an elementary volume with respect to time is given by:

$$
d x(h(x, t+d t)-h(x, t)) d t=d t(h<u>(x+d x, t)+h<u>(x, t))-\dot{E} d t d x
$$

Thus:

$$
\begin{gathered}
\frac{\partial h}{\partial t} d x d t=-d t d x \frac{\partial}{\partial x}(h<u>)-\dot{E} d t d x \\
\dot{E}+\frac{\partial h}{\partial t}=\frac{\partial}{\partial t}\left(h_{\infty}-h(x, t)\right) \\
\frac{\partial}{\partial t}\left(h_{\infty}-h(x, t)\right)=\frac{\partial}{\partial x}\left(\frac{h^{3}}{3 \eta}\left[\gamma \frac{d^{3} h}{d x^{3}}-\rho g \frac{d h}{d x}\right]\right)
\end{gathered}
$$


Front position determination (ImageJ)

ImageJ was used in order to determine the compaction front position. The photograph is first converted into a grey scale image. Then, the intensity along a diameter (for the circular geometry) or along an axis of symmetry (for the rectangular deposit) is computed. An example of such a profile is given in Fig S1 below. From this profile, we determine manually the two compaction fronts (denoted by arrows in Fig S1).
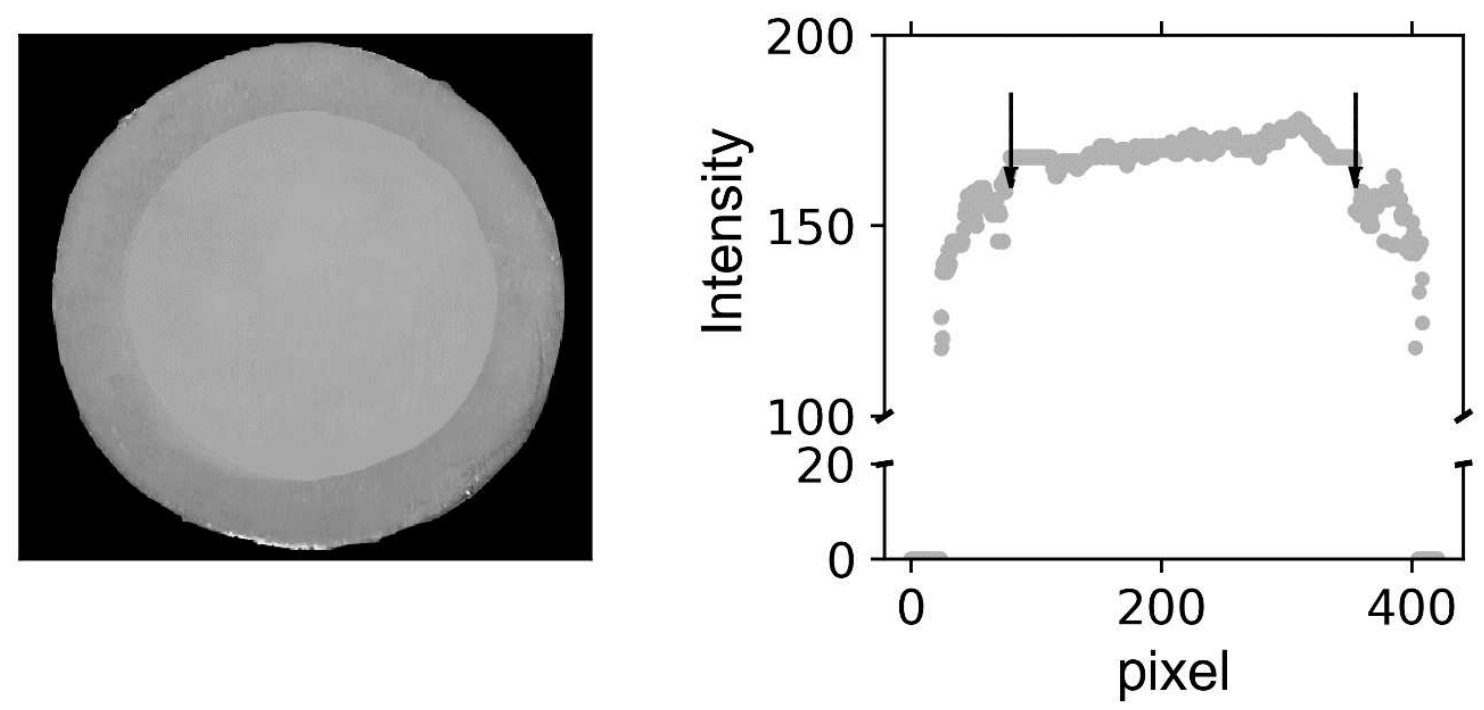

Fig. S1 Example of compaction front position determination using the ImageJ software. Photograph of the circular sample (diameter $4 \mathrm{~cm}$ ) converted into grey levels (left). Grey level along a diameter (right). Slope changes, indicated by arrows, correspond to compaction fronts. Let us remind the reader that water fronts could not be followed in this work because they corresponded to the apparition of cracks. 
Sketch of the principle of the optical profilometry used in this work

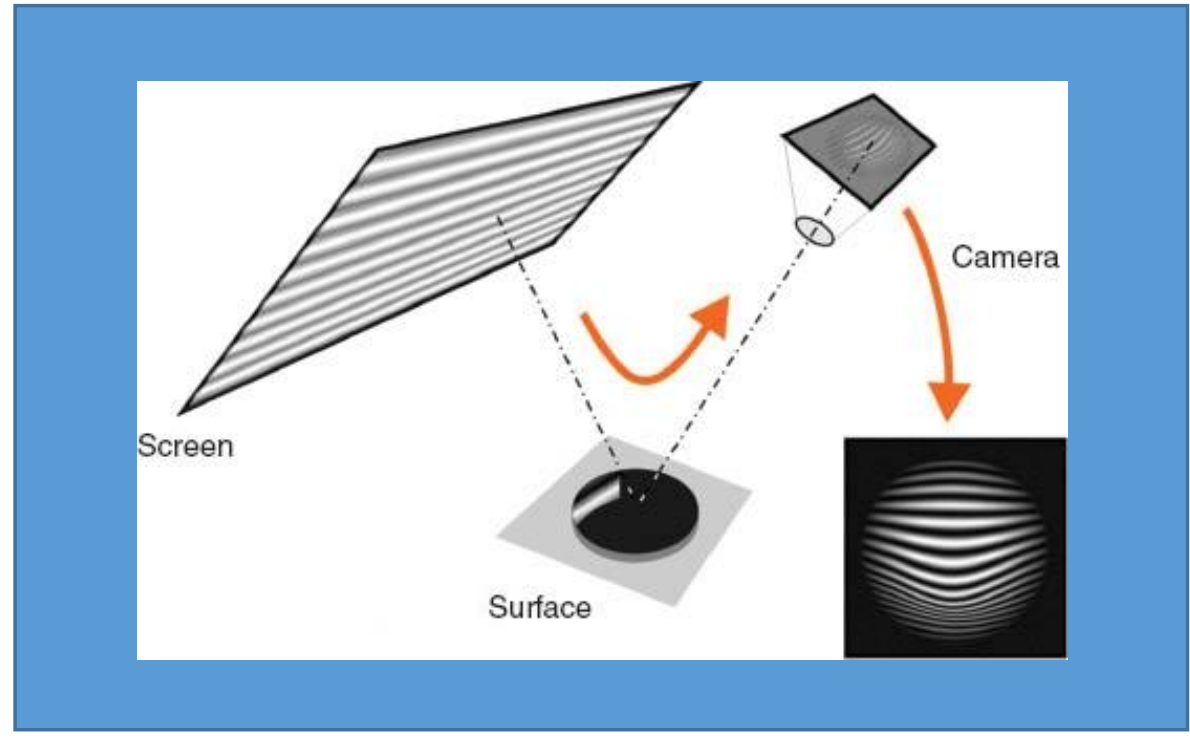

A light source projects the image of a grid onto the sample. A CCD camera captures images of this grid reflected by the surface of the suspension. The non-planar surface of the drying film creates a distortion of the image of the grid. An image processing software transforms the deformed grid into quantified information of the local slopes of the surface as well as XYZ coordinate to reconstruct the topography. 
Routh and Russel model
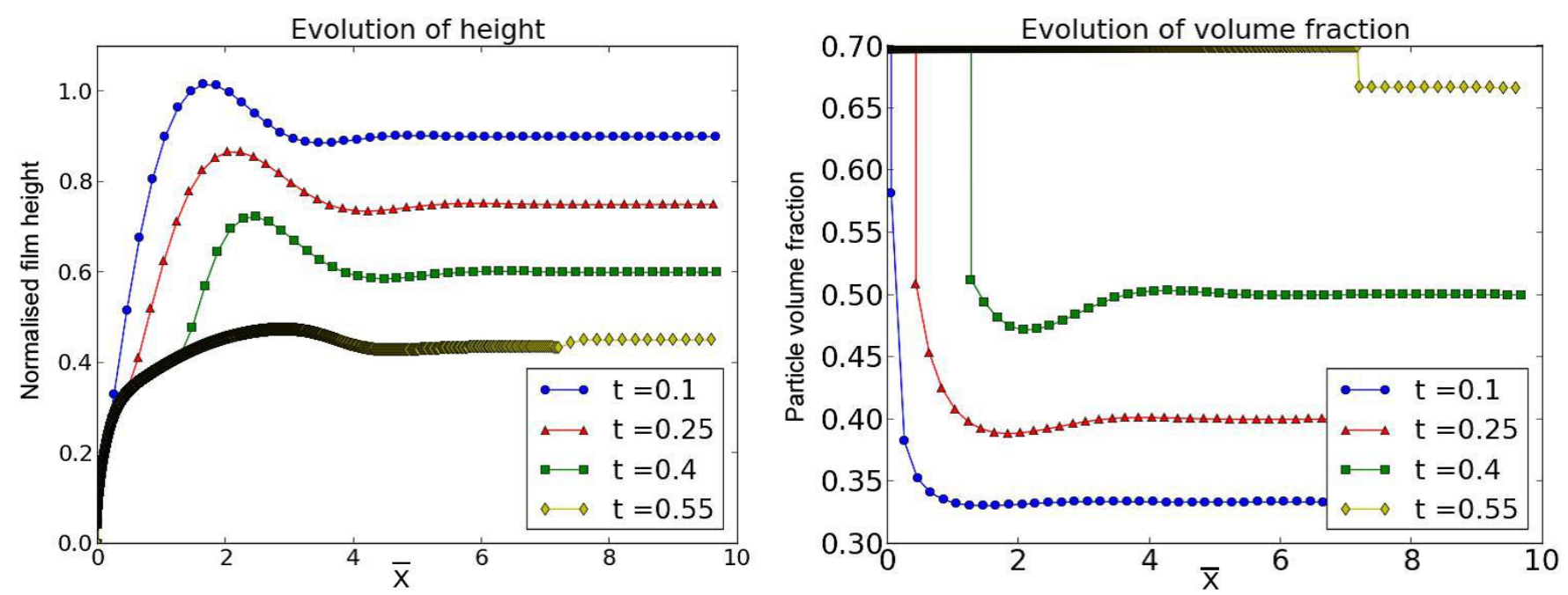

Fig. S2 Film profile (left) and particle volume fraction (right) versus lateral dimension at different times during drying calculated using our cellular simulation in the conditions of the Routh and Russel (RR) model. Film height scaled on the initial film thickness, lateral dimension (X) scaled on the capillary length in the RR sense, time scaled on the characteristic time $\frac{H_{0}}{\dot{E}}$. Initial particle volume fraction: 0.3. Volume fraction at close packing: 0.7. Infinite capillary pressure.

Contribution of hydrostatic pressure

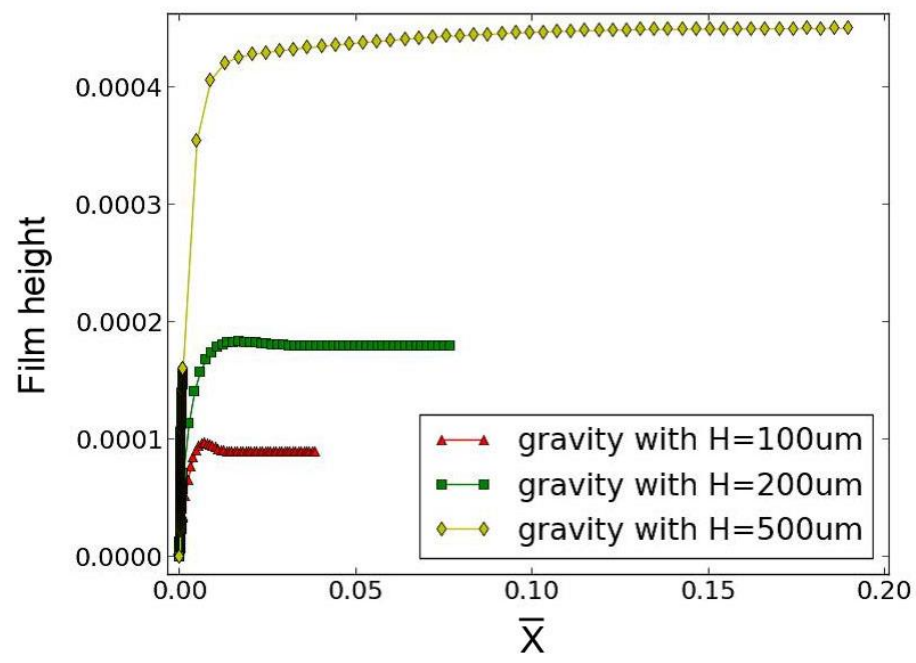

Fig. S3 Film profile versus lateral dimension at time $=0.1$ for different initial thicknesses (i.e. different $L_{c a p} / L_{g}$ ratios) when taking hydrostatics into account. Film height and lateral dimension $(\mathrm{X})$ in real dimension $(\mathrm{m})$. time scaled on the characteristic time $\frac{H_{0}}{\dot{E}} . L_{c a p} / L_{g}=1.5$; 3; 7.5 for $H_{0}=100 \mu \mathrm{m} ; 200 \mu \mathrm{m} ; 500 \mu \mathrm{m}$, respectively. $\gamma=70 \mathrm{~mJ} / \mathrm{m}^{2}, \eta_{0}=1 \mathrm{~Pa} . \mathrm{s}, \dot{E}=10^{-8} \mathrm{~m} / \mathrm{s}$, $\rho=1000 \mathrm{~kg} / \mathrm{m}^{3}, \mathrm{~g}=9.81 \mathrm{~m} / \mathrm{s}^{2}$. Initial particle volume fraction: 0.3 . Volume fraction at close packing: 0.7 . Infinite capillary pressure (taken at 1000 for calculation). 
Particle front position vs time with hydrostatics

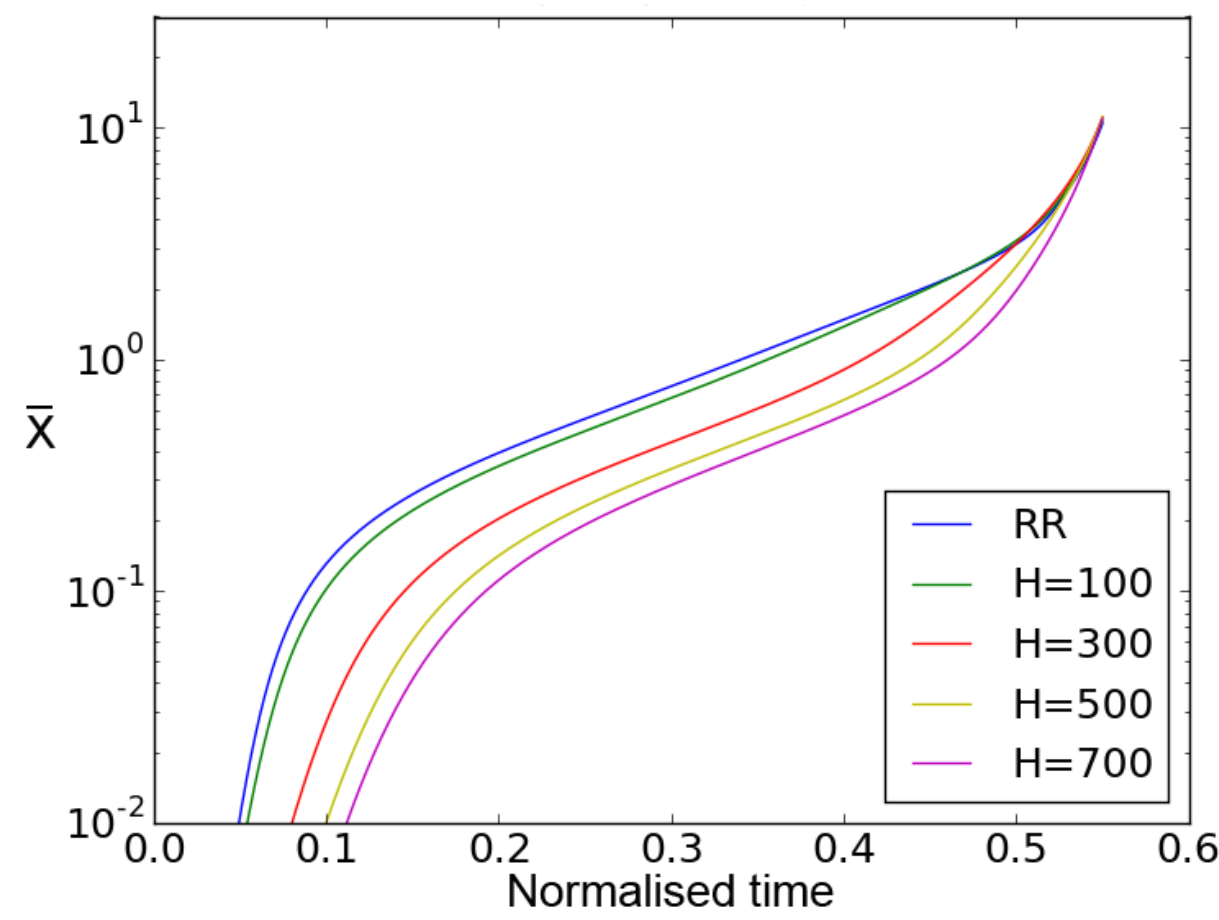

Fig. S4 Particle front position versus time for different initial film thicknesses when taking hydrostatics into account. Comparison with RR model (blue curve). Lateral dimension (X) scaled on $L_{c a p}$, time scaled on $\frac{H_{0}}{\dot{E}}$. Other parameters like in Fig. S2. 


\section{Taylor diffusion of suspensions in a semi-parabolic flow}

The hydrodynamics of drying suspensions is commonly treated in the lubrication approximation. This approximation for Newtonian fluids consists in a locally parabolic velocity profile driven by some pressure gradient. It is analogue to some extent to Poiseuille parabolic flows occurring in pipes of cylindrical section at low Reynolds numbers. When considering the longitudinal diffusion of a solute or suspended particles (i.e. parallel to the flow direction) one may be concerned by the fact that the velocity field is not uniform. Suspended particles will be advected faster in the regions close to the free boundary than at the vicinity of the solid surface. Such an heterogeneous advection is known as the Taylor, or Taylor-Aris diffusion problem and has received a satisfactory solution in the case of laminar Poiseuille flows occurring in cylindrical pipes $[1,2]$. We outline below an equivalent treatment in the presence of a semiparabolic Poiseuille lubrication flow, and for a concentration independent diffusion coefficient $D$.

The lubrication flow In the case of drying suspensions, there are two boundary conditions : vanishing velocity at the solid surface and vanishing stress (vanishing velocity gradient) at the free air fluid surface. If one denotes by $y$ the direction normal and $x$ the direction parallel to the surface, the resulting flow displays a semi-parabolic velocity profile

$$
\boldsymbol{u}=u(x, y) \boldsymbol{e}_{x}=\frac{(y-2 h) y}{2 \eta_{0}} \frac{\partial p_{h}}{\partial x}(x) \boldsymbol{e}_{x}
$$

$p_{h}(x)$ being the total hydrostatic pressure with no $y$ dependence, $\eta_{0}$ the dynamic viscosity of the suspension (solvent and particles), $h$ the film thickness.

The local velocity field can also be expressed by means of the average cross-section velocity, obtained by applying a projection operator $\mathcal{P}$

$$
\mathcal{P}(u)=\mathcal{P} u=\frac{1}{h} \int_{0}^{h} \mathrm{~d} y u(x, y) .
$$

$\mathcal{P}$ is a linear operator mapping any function of $x, y$ onto a function of $x$ only, and obeys the standard projection rule $\mathcal{P}(\mathcal{P})=\mathcal{P}^{2}=\mathcal{P}$. Then, in the lubrication approximation,

$$
u(x, y)=\frac{3 y(2 h-y)}{2 h^{2}} \mathcal{P} u(x)
$$

The lubrication approximation is expected to hold if the velocity gradient along the $x$ direction are small compared with the vertical gradient, and the time scales of the problem are long compared with $\tau_{h}=\rho H^{2} / \eta_{0}, \rho$ mass density of the suspension, $H \sim h$ typical height, which is the characteristic time for stabilizing a Poiseuille parabolic velocity profile.

The transport of a species in the presence of a stream involves explicitly the velocity field $\boldsymbol{u}$. In the case of an incompressible flow accross a constant section cylindrical pipe, the velocity gradients have the effect of enhancing the diffusion constant of the species along the longitudinal direction.

Projection of the transport equations Our notations will be the following ones :

- commas stand for partial differentiation, e.g. $f(x, y, t)_{, x}=\partial f / \partial x$.

- $\mathcal{P}$ is the vertical average projection operator, and $Q=1-\mathcal{P}$ its orthogonal complement. 
With these notations, one checks the following properties

- $(\mathcal{P} f)_{, t}=\mathcal{P}\left(f_{, t}\right)$ and $(\mathcal{Q} f)_{, t}=\mathcal{Q}\left(f_{, t}\right)$,

- $(\mathcal{P} f)_{, x}=\mathcal{P}\left(f_{, x}\right)$ and $(\mathcal{Q} f)_{, x}=\mathcal{Q}\left(f_{, x}\right)$,

- $\mathcal{P}(g f)=g \mathcal{P}(f)$ if $g$ is function of $x$ only,

- $\mathcal{P} \mathcal{Q} f=Q \mathcal{P} f=0$,

- $\mathcal{P}\left(f_{, y}\right)=\frac{f(y=h)-f(y=0)}{h}$,

- $\mathcal{P}\left(f_{, y y}\right)=\frac{f_{, y}(y=h)-f_{, y}(y=0)}{h}$,

- $(\mathcal{P} f)_{, y}=0$,

- $(2 f)_{, y}=f_{, y}$

It is expected as a rule that $Q f$ remains smaller than $\mathcal{P} f$, and expansions are going to be performed according to this rationale. We assume that the velocity field $u$ and the elevation $h$ are constant and uniform. The starting point is the collective diffusion equation

$$
\Phi_{, t}+u \Phi_{, x}=D \Phi_{, x x}+D \Phi_{, y y}
$$

This equation expresses the conservation of the latex suspension volume fraction $\Phi$, itself proportionnal to the particle number density. Gradients of $\Phi$ create a current $-D \nabla \Phi$ which involves the diffusion coefficient $D$.

The first step is to apply $\mathcal{P}$ to eq. (4). The result is

$$
\mathcal{P} \Phi_{, t}+\mathcal{P}\left(u \Phi_{, x}\right)=\mathcal{P}\left(D \Phi_{, x}\right)_{, x}
$$

where the second term in r.h.s vanishes due to $\Phi_{, y}(y=0)=0, \Phi_{, y}(y=h)=0$, and one has

$$
\mathcal{P} \phi_{, t}+\mathcal{P} u \mathcal{P} \Phi_{, x}+\mathcal{P}\left(u \mathcal{Q} \Phi_{, x}\right)=D \mathcal{P} \Phi_{, x x}
$$

Similar considerations guide the orthogonal projection of the transport equation. The idea is to substract eq.(6) from eq.(4).

$$
2 \phi_{, t}+\mathcal{Q} u \mathcal{P} \Phi_{, x}+\mathcal{Q}\left(u \mathcal{Q} \Phi_{, x}\right)=D(Q \Phi)_{, y y}+D(Q \Phi)_{, x x}
$$

Terms in (6) and (7) are not of the same magnitude and must be hierarchized. In order to proceed in a systematic way, it is now necessary to guess which are the relevant scales of the problem and to rescale both equations appropriately.

Rescaling and effective equations Let us assume that vertical lengths scale as $H$ and define $\bar{y}=y / H, \bar{h}=h / H$, that horizontal lengths scale as $L_{\phi}$ and define $\bar{x}=x / L_{\phi}$. One introduces a (yet undetermined) characteristic time scale for horizontal diffusion $\tau_{D}\left(\bar{t}=t / \tau_{D}\right)$ and a velocity scale $V$ representative of the stream $u$, with $\bar{u}=u / V$.

From $V, D$ and $L_{\phi}$ can be derived a horizontal Peclet number $\mathrm{Pe}_{h}=V L_{\phi} / D$. An interesting limit emerges when $L_{\phi}$ is larger than $H$, with the effect of inhomogeneous flow resulting in the redefinition of the diffusion constant, and the possibility to derive a simple, effective 1d-diffusion equation. We therefore assume $H / L_{\phi} \ll 1$.

The two projected equations rescale as

$$
\begin{aligned}
\frac{1}{\tau_{D}} \mathcal{Q} \phi_{, \bar{t}}+\frac{V}{L_{\phi}} \mathcal{Q} \overline{\mathcal{P}} \Phi_{, \bar{x}}+\frac{V}{L_{\phi}} \mathcal{Q}\left(\bar{u} \mathcal{Q} \Phi_{, \bar{x}}\right) & =\frac{D}{H^{2}}(Q \Phi)_{, \overline{y y}}+\frac{D}{L_{\phi}^{2}}(Q \Phi)_{, \overline{x x}} \\
\frac{1}{\tau_{D}} \mathcal{P} \phi_{, \bar{t}}+\frac{V}{L_{\phi}} \mathcal{P} \bar{u} \mathcal{P} \Phi_{, \bar{x}}+\frac{V}{L_{\phi}} \mathcal{P}\left(\bar{u} \mathcal{Q} \Phi_{, x}\right) & =\frac{D}{L_{\phi}^{2}} \mathcal{P} \Phi_{, \overline{x x}}
\end{aligned}
$$


The usual treatment of the Taylor diffusion assumes that the second term of lhs and the first term of rhs balance in eq. (8), while other terms are subdominant. Introducing $\varepsilon=\frac{H^{2} V}{D L_{\phi}}$, one finds

$$
\begin{aligned}
\frac{L_{\phi}^{2}}{D \tau_{D}} \frac{H^{2}}{L_{\phi}^{2}} \mathcal{Q} \phi_{, \bar{t}}+\varepsilon \mathcal{Q} \overline{\mathcal{P}} \mathcal{P} \Phi_{, \bar{x}}+\varepsilon \mathcal{Q}\left(\bar{u} \mathrm{Q} \Phi_{, \bar{x}}\right) & =(Q \Phi)_{, \overline{y y}}+\frac{H^{2}}{L_{\phi}^{2}}(Q \Phi)_{, \overline{x x}} \\
\frac{L_{\phi}^{2}}{D \tau_{D}} \mathcal{P} \phi_{, \bar{t}}+\mathrm{Pe}_{h} \mathcal{P} \bar{u} \mathcal{P} \Phi_{, \bar{x}}+\operatorname{Pe}_{h} \mathcal{P}\left(\bar{u} Q \Phi_{, x}\right) & =\mathcal{P} \Phi_{, \overline{x x}}
\end{aligned}
$$

One expects $\varepsilon \ll 1, Q \Phi \sim \varepsilon$ and $Q \Phi \ll \mathcal{P} \Phi$. The horizontal diffusion time scale is set by the equivalent $L_{\phi}^{2} / D \tau_{D} \sim 1$, which entails $H^{2} / D \tau_{D} \ll 1$. The primary balance of the first equation reads

$$
\varepsilon \mathcal{Q} \overline{\mathcal{P}} \Phi_{, \bar{x}}=(\mathcal{Q} \Phi)_{, \overline{y y}}
$$

Recast in the original, unscaled variables, one finds

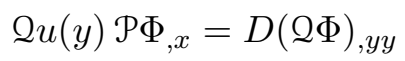

As $Q u$ is a known function of $y$, the above equation reduces to a double quadrature accompanied by the boundary conditions $\Phi_{, y}=(Q \Phi)_{, y}=0$ at $y=h$ and $y=0$, and the integral condition $\mathcal{P} Q \Phi=0$. The solution reads

$$
\begin{aligned}
\mathcal{Q} u & =\left(-1+\frac{3 y}{h}-\frac{3}{2}\left(\frac{y}{h}\right)^{2}\right) \mathcal{P} u \\
\mathcal{Q} \Phi & =\frac{h^{2} \mathcal{P} \Phi_{, x} \mathcal{P} u}{8 D}\left(\frac{8}{15}-4 \frac{y^{2}}{h^{2}}+4 \frac{y^{3}}{h^{3}}-\frac{y^{4}}{h^{4}}\right) .
\end{aligned}
$$

Then, one can substitute for the $\mathcal{P}\left(u Q \Phi_{, x}\right)$ term in eq. (17)

$$
\begin{aligned}
u \mathcal{Q} \Phi_{, x} & =\frac{3 y(2 h-y)}{2 h^{2}} \times\left(\frac{8}{15}-4 \frac{y^{2}}{h^{2}}+4 \frac{y^{3}}{h^{3}}-\frac{y^{4}}{h^{4}}\right) \times \frac{h^{2} \mathcal{P} \Phi_{, x x} \mathcal{P} u^{2}}{8 D} \\
\mathcal{P}\left(u \mathcal{Q} \Phi_{, x}\right) & =-\frac{2}{105} \frac{h^{2} \mathcal{P} \Phi_{, x x} \mathcal{P} u^{2}}{D}
\end{aligned}
$$

leading eventually to

$$
\begin{aligned}
\mathcal{P} \phi_{, t}+\mathcal{P} u \mathcal{P} \Phi_{, x} & =\left[D+\frac{2(h \mathcal{P} u)^{2}}{105 D}\right] \mathcal{P} \Phi_{, x x} \\
\mathcal{P} \phi_{, \bar{t}}+\operatorname{Pe}_{h} \mathcal{P} \bar{u} \mathcal{P} \Phi_{, \bar{x}} & =\left[1+\varepsilon \operatorname{Pe}_{h} \frac{2(\bar{h} \mathcal{P} \bar{u})^{2}}{105}\right] \mathcal{P} \Phi_{\overline{x x}}
\end{aligned}
$$

It results in an effective enhanced 1-d diffusion coefficient $D_{\text {eff }}=D+2 h^{2}(\mathcal{P} u)^{2} / 105 D$, known as Taylor diffusion constant.

Discussion It is therefore possible to integrate out the coupled effect of lateral diffusion and lateral shear velocity drift and to obtain an effective $1 d$ transport equation. This can only be done properly if the longitudinal scale $L_{\phi}$ of the concentration gradient is larger than the thickness of the film $H$. At large horizontal Peclet number, there is the possibility of a significant enhancement of the effective diffusion constant. 
The previous derivation is valid under the assumptions $H / L_{\phi} \ll 1, H^{2} V / D L_{\phi} \ll 1$, $\tau_{D} \sim L_{\phi}^{2} / D$.

At the vicinity of the solid (wet gel front) region, the flow has to switch from parabolic to uniform. This is also a region where strong concentration gradients are expected. More likely than not, the above assumptions will break down at the vicinity of the solid front. A comprehensive approach would therefore be needed to deal with a truly $2 \mathrm{~d}$ problem in variables $x$ and $y$.

\section{Références}

[1] Geoffrey Taylor. Dispersion of soluble matter in solvent flowing slowly through a tube. Proceedings of the Royal Society of London A : Mathematical, Physical and Engineering Sciences, 219(1137) :186-203, 1953.

[2] R. Aris. On the dispersion of a solute in a fluid flowing through a tube. Proceedings of the Royal Society of London A : Mathematical, Physical and Engineering Sciences, 235(1200):67-77, 1956. 